\title{
The Influence of Radio-Frequency Transmit Field Inhomogeneities on the Accuracy of G-ratio Weighted Imaging
}

Tim M. Emmenegger 1,2, Gergely David ${ }^{1,2}$, Mohammad Ashtarayeh ${ }^{2}$, Francisco J. Fritz ${ }^{2}$, Isabel Ellerbrock ${ }^{2,3}$, Gunther Helms ${ }^{4}$, Evelyne Balteau ${ }^{5}$, Patrick Freund ${ }^{1,6,7}$ and Siawoosh Mohammadi2,6*

' Spinal Cord Injury Center Balgrist, University Hospital Zurich, University of Zurich, Zurich, Switzerland, ${ }^{2}$ Department of Systems Neuroscience, University Medical Center Hamburg-Eppendorf, Hamburg, Germany, ${ }^{3}$ Department of Clinical Neuroscience, Karolinska Institutet, Stockholm, Sweden, ${ }^{4}$ Medical Radiation Physics, Clinical Sciences Lund (IKVL), Lund University, Lund, Sweden, ${ }^{5}$ GIGA Institute, University of Liège, Liège, Belgium, ${ }^{6}$ Department of Neurophysics, Max Planck Institute for Human Cognitive and Brain Sciences, Leipzig, Germany, ${ }^{7}$ Wellcome Trust Centre for Neuroimaging, University College London, London, United Kingdom

\section{OPEN ACCESS}

Edited by:

Tim B. Dyrby,

Technical University of Denmark,

Denmark

Reviewed by:

Viktor Vegh,

The University of Queensland,

Australia

Olivier Commowick, Inria Rennes-Bretagne Atlantique

Research Centre, France

${ }^{*}$ Correspondence: Siawoosh Mohammadi s.mohammadi@uke.de

Specialty section:

This article was submitted to Brain Imaging Methods,

a section of the journal Frontiers in Neuroscience

Received: 01 March 2021 Accepted: 01 June 2021 Published: 05 July 2021

Citation:

Emmenegger TM, David G, Ashtarayeh M, Fritz FJ, Ellerbrock I, Helms G, Balteau E, Freund P and Mohammadi S (2021) The Influence of Radio-Frequency Transmit Field Inhomogeneities on the Accuracy

of G-ratio Weighted Imaging.

Front. Neurosci. 15:674719.

doi: 10.3389/fnins.2021.674719
G-ratio weighted imaging is a non-invasive, in-vivo MRI-based technique that aims at estimating an aggregated measure of relative myelination of axons across the entire brain white matter. The MR g-ratio and its constituents (axonal and myelin volume fraction) are more specific to the tissue microstructure than conventional MRI metrics targeting either the myelin or axonal compartment. To calculate the MR g-ratio, an MRI-based myelin-mapping technique is combined with an axon-sensitive MR technique (such as diffusion MRI). Correction for radio-frequency transmit $(\mathrm{B} 1+$ ) field inhomogeneities is crucial for myelin mapping techniques such as magnetization transfer saturation. Here we assessed the effect of $\mathrm{B} 1+$ correction on g-ratio weighted imaging. To this end, the B1+ field was measured and the B1+ corrected MR g-ratio was used as the reference in a Bland-Altman analysis. We found a substantial bias $(\approx-$ $89 \%)$ and error $(\approx 37 \%)$ relative to the dynamic range of g-ratio values in the white matter if the B1+ correction was not applied. Moreover, we tested the efficiency of a data-driven $\mathrm{B} 1+$ correction approach that was applied retrospectively without additional reference measurements. We found that it reduced the bias and error in the MR g-ratio by a factor of three. The data-driven correction is readily available in the open-source hMRI toolbox (www.hmri.info) which is embedded in the statistical parameter mapping (SPM) framework.

Keywords: myelin volume fraction, axon volume fraction, radio-frequency transmit field inhomogeneities, $\mathrm{B}_{1}+$ correction, multi-parameter mapping, diffusion MRI, magnetization transfer saturation, MR g-ratio

\section{INTRODUCTION}

The g-ratio [i.e., the ratio between the inner ( $\mathrm{r}$ ) and outer $(\mathrm{R})$ radius of an axon with myelin sheath $(\mathrm{g}$-ratio $=\mathrm{r} / \mathrm{R}$ )] of a given axon quantifies the degree of relative myelination, ranging between 0 (no axon) and 1 (no myelin). The g-ratio captures both axonal and myelin damage by incorporating axonal and myelin volumes in one metric, making it potentially more specific to tissue integrity than 
focusing on one of these aspects only. For example, in multiple sclerosis, the g-ratio increases if the underlying disease mechanism is solely driven by demyelination (Yu et al., 2019), but is expected to remain unaffected if demyelination is accompanied by axonal degeneration. To differentiate such processes and understand their functional implications, neuroscience and clinical research would greatly benefit from in-vivo whole-brain measurements of MR g-ratio. Until recently, the g-ratio was measurable only by means of histology (Hildebrand and Hahn, 1978), which restricted the analyses to a small number of axons and a limited number of small brain regions or pathways. Stikov et al. $(2011,2015)$ introduced a methodology for an MRI-based whole-brain "aggregate" g-ratio mapping, to which we refer as "MR g-ratio" or "g-ratio weighted imaging." In g-ratio weighted imaging, the MR g-ratio is computed on a voxel-by-voxel basis from the axonal (AVF) and myelin volume fraction (MVF) maps and reflects a weighted mean of g-ratio values within the voxel (West et al., 2016). Therefore, g-ratio weighted imaging requires the acquisition of separate sets of images that are sensitive to AVF and MVF, respectively (Campbell et al., 2018; Mohammadi and Callaghan, 2020). To generate MVF and AVF from the measured MR parameters, a calibration step is required that converts the measured MR-visible water signals into the respective volume fractions (Mohammadi and Callaghan, 2020).

Magnetization transfer saturation $\left(\mathrm{MT}_{\text {sat }}\right)$ has often been used as proxy for MVF (Mohammadi et al., 2015) as it is minimally affected by the longitudinal relaxation time (Helms et al., 2008) and is expected to show high correlation with macromolecular content (Sereno et al., 2013; Callaghan et al., 2015a; Campbell et al., 2018), making it a sensitive metric of MVF. One common approach to estimate AVF complements the parameters from neurite orientation and dispersion density imaging (NODDI Zhang et al., 2012) with a MVF-proxy, e.g., $\mathrm{MT}_{\text {sat }}$ (Ellerbrock and Mohammadi, 2018; Kamagata et al., 2019), to correct for the missing myelin water signal in diffusion MRI measurements (Stikov et al., 2015). Maps of $\mathrm{MT}_{\text {sat }}$ can be obtained, among others, from the multi-parameter mapping (MPM) protocol (Weiskopf et al., 2013) in combination with the hMRI toolbox ${ }^{1}$ (Callaghan et al., 2019; Tabelow et al., 2019).

Although the $\mathrm{MT}_{\text {sat }}$ measure is largely insensitive to transmit field $\left(\mathrm{B}_{1}+\right)$ inhomogeneities (Helms et al., 2008), it still shows a residual dependence which introduces a bias and/or error in the $\mathrm{MT}_{\text {sat }}$ maps that can propagate into the MR g-ratio and lead to systematic bias. Such $\mathrm{B}_{1}+$ inhomogeneities can be corrected based on an independently acquired $\mathrm{B}_{1}+$ field map measurement (Helms, 2015; Helms et al., 2021). Residual $\mathrm{B}_{1}+$ inhomogeneity effects on $\mathrm{MT}_{\text {sat }}$ have been shown to be not negligible when the $\mathrm{B}_{1}+$ correction was omitted (Helms, 2015; Helms et al., 2021). However, the impact of $\mathrm{B}_{1}+$ correction on MR g-ratio estimates is unknown. Additionally, it is unclear whether these residual $\mathrm{B}_{1}+$ inhomogeneity in $\mathrm{MT}_{\text {sat }}$ and the MR g-ratio can retrospectively be corrected using a data-driven $\mathrm{B}_{1}+$ field inhomogeneities estimation approach such as the "unified segmentation based correction of $\mathrm{R} 1$ maps for $\mathrm{B}_{1}+$ inhomogeneities" (UNICORT, (Weiskopf et al., 2011)).

\footnotetext{
${ }^{1}$ www.hMRI.info
}

In this study, we investigate the effect of $\mathrm{B}_{1}+$ inhomogeneities on MR g-ratio maps when omitting the $\mathrm{B}_{1}+$ correction. As a reference, we use the $\mathrm{B}_{1}+$ corrected $\mathrm{MR}$ g-ratio from a dataset of healthy controls. We compare the reference MR g-ratio values against (i) values obtained without $\mathrm{B}_{1}+$ correction and (ii) values obtained with $\mathrm{B}_{1}+$ correction using the data-driven UNICORT approach.

\section{MATERIALS AND METHODS}

\section{Subjects}

This study included 25 healthy control subjects (12 females, age (mean \pm standard deviation) of $25.4 \pm 2.4$ years). They were recruited at the University Medical Centre HamburgEppendorf and screened for neurological or psychiatric illness. The study was in agreement with the Declaration of Helsinki and was approved by the local ethics committee (Ärztekammer Hamburg \#PV5141).

\section{Data Acquisition}

Each subject was scanned twice within 1 week in a whole-body 3T Tim TRIO MR scanner (Siemens Healthcare, Erlangen, Germany) using the body RF-coil for transmission and a 32channel radiofrequency ( $R F)$ head coil for signal reception, respectively. The MR acquisition on both scan days included a multi-parameter mapping (MPM) (Weiskopf et al., 2013; Callaghan et al., 2015b) and a diffusion-weighted imaging (DWI) protocol. The MPM protocol consists of three differently weighted 3D-multi-echo spoiled gradient echo sequences (Siemens FLASH). The echo train length and flip angle for the proton density (PD) weighted, T1-weighted, and magnetization transfer (MT) weighted sequences were $8 / 6,8 / 21$, and $6 / 6^{\circ}$, respectively. The MT-weighted sequence had a Gaussian RF pulse ( $2 \mathrm{kHz}$ off resonance with $4 \mathrm{~ms}$ duration and a nominal flip angle of $220^{\circ}$ ). All other sequence parameters were the same for the three sequences: repetition time (TR) $25 \mathrm{~ms}$, echo spacing, resolution $0.8 \mathrm{~mm}$ isotropic; field of view (FoV) $166 \times 224 \times$ $256 \mathrm{~mm}^{3}$, readout bandwidth $488 \mathrm{~Hz} /$ pixel, partially parallel imaging using the GRAPPA algorithm was employed in each phase-encoded direction (anterior-posterior and right-left) with 40 reference lines and a speed up factor of two, total acquisition time: $\sim 25 \mathrm{~min}$. The $\mathrm{B}_{1}+$ field reference map was acquired using the three-dimensional echo-planar imaging (3D EPI) method, including field maps for distortion correction (Lutti et al., 2010).

The DWI sequence was a twice-refocused single-shot spinecho EPI scheme (Reese et al., 2003), consisting of 12 nondiffusion-weighted images ( $b_{0}$ images), equidistantly distributed across the diffusion weighted images. The diffusion-weighted images were acquired at two $b$-values $\left(1000 \frac{\mathrm{s}}{\mathrm{mm}^{2}}\right.$ and $\left.2000 \frac{\mathrm{s}}{\mathrm{mm}^{2}}\right)$, sampled along 60 unique diffusion-gradient directions within each shell. The entire protocol was repeated with identical parameters but with reversed phase encoding direction (anteriorposterior) to correct for susceptibility-related image distortions (blip-up, blip-down correction). In total, 264 images were acquired per subject (120 diffusion-weighted images, $12 b_{0}$ images, each acquired twice). Other acquisition parameters were: 
86 slices with no gap, $\mathrm{TR}=7.1 \mathrm{~s}, \mathrm{TE}=122 \mathrm{~ms}$, an isotropic voxel size of $(1.6 \mathrm{~mm})^{3}, \mathrm{FoV}=224 \times 224 \times 138 \mathrm{~mm}^{3}, 7 / 8$ partial Fourier imaging in phase encoding direction, readout bandwidth. To accelerate the data acquisition, GRAPPA (inplane acceleration with factor two) and simultaneous multi-slice acquisitions ("multiband," slice acceleration factor two) (Feinberg et al., 2010; Moeller et al., 2010; Xu et al., 2013) were used as described in Setsompop et al. (2012). The image reconstruction algorithm was provided by the University of Minnesota Centre for Magnetic Resonance Research. The total acquisition time was $\sim 37 \mathrm{~min}$.

\section{Data Processing}

$\mathrm{MT}_{\text {sat }}$ maps were generated in the SPM-based hMRI toolbox (Tabelow et al., 2019). Note that the hMRI toolbox also generates additional maps of longitudinal $\left(\mathrm{R}_{1}\right)$ and effective transverse relaxation rates $\left(R_{2}^{\star}\right)$ and $P D$. Three $M_{\text {sat }}$ maps were generated: (i) $\mathrm{MT}_{\text {sat }}^{\mathrm{NO}}$ maps, without $\mathrm{B}_{1}+$ correction; (ii) $\mathrm{MT}_{\text {sat }}^{\mathrm{B} 1}$ map, using the reference $\mathrm{B}_{1}+$ field map for correction (Lutti et al., 2010); and (iii) $\mathrm{MT}_{\text {sat }}^{\mathrm{UN}}$ maps, using the datadriven UNICORT approach for $\mathrm{B}_{1}+$ estimation (Weiskopf et al., 2011; see Supplementary Figure 2). UNICORT is a probabilistic framework for unified-segmentation based correction of $\mathrm{R}_{1}$ maps for $\mathrm{B}_{1}+$ inhomogeneities. The framework incorporates a physically informed generative model of smooth $\mathrm{B}_{1}+$ inhomogeneities and their multiplicative effect on $R_{1}$ estimates (Weiskopf et al., 2011). Parameters used in UNICORT such as the smoothness and regularization were optimized for $\mathrm{R}_{1} \mathrm{~B}_{1}+$ correction in a $3 \mathrm{~T}$ scanner (i.e., Tim Trio scanner-Weiskopf et al., 2011).

For $\mathrm{B}_{1}+$ correction, we used the following heuristic correction factor as detailed in Helms (2015), and Helms et al. (2021):

$$
\mathrm{MT}_{\text {sat }}^{\mathrm{Corr}}=\mathrm{MT}_{\text {sat }}^{\mathrm{NO}} \frac{1-C}{1-\mathrm{CB}_{1}^{+}}
$$

where $C$ has been calibrated to be 0.4 for the MT pulse used in this paper. $\mathrm{B}_{1}+$ can be either measured $\left(\mathrm{MT}_{\text {sat }}^{\mathrm{Corr}}=\mathrm{MT}_{\text {sat }}^{\mathrm{B} 1}\right)$ or estimated with the UNICORT approach $\left(\mathrm{MT}_{\text {sat }}^{\mathrm{Cor}}=\mathrm{MT}_{\text {sat }}^{\mathrm{UN}}\right)$.

The DWI data were processed based on the pipeline described in Ellerbrock and Mohammadi (2018) using the SPM-based ACID toolbox ${ }^{2}$. It included several artifact corrections such as Rician signal bias correction (i.e., denoising) (André et al., 2014), correction for eddy current and motion artifacts (Mohammadi et al., 2010, 2014), and correction for image distortions due to susceptibility artifact using reversed phase encoding (Ruthotto et al., 2012, 2013; Macdonald and Ruthotto, 2018). The corrected images were fitted with the NODDI signal model (Zhang et al., $2012)$ to estimate the intra-cellular volume fraction $\left(\nu_{\text {icvf }}\right)$, the isotropic volume fraction $\left(v_{\text {iso }}\right)$, and the orientation dispersion index (ODI) in each voxel.

${ }^{2}$ http://www.diffusiontools.com

\section{Spatial Alignment Co-registration}

The voxel-wise arithmetic between the $\mathrm{MT}_{\text {sat }}$ and $\nu_{\text {icvf }}$ maps, necessary for MR g-ratio computation, requires an accurate spatial alignment between the two maps (Mohammadi et al., 2015). To this end, we created two white matter (WM) tissue probability maps (TPMs) based on the ODI and $\mathrm{MT}_{\text {sat }}^{\mathrm{B} 1}$ maps, respectively (Figure 1). To reduce the influence of contrast-specific artifacts (e.g., due to subject motion) on the registration quality, the WM TPM of the ODI map was coregistered to the WM TPM of the $\mathrm{MT}_{\text {sat }}^{\mathrm{B} 1}$ map using rigid-body registration (spm_coreg algorithm, SPM toolbox). The estimated transformation parameters were applied to all other NODDI maps as well. Note that the segmentation quality of the second session was unsatisfactory for two subjects, and the $\mathrm{R}_{1}^{\mathrm{B}_{1}}$ map $\left(\mathrm{R}_{1}\right.$ with $\mathrm{B}_{1}+$ inhomogeneities bias correction using the $\mathrm{B}_{1}+$ reference measurements) was used to generate the WM TPM instead. In another subject, the $v_{\text {iso }}$ was segmented instead of the ODI to achieve satisfactory WM segments.

\section{Normalization}

Spatial normalization was performed in four steps. First, a rough alignment of the $\mathrm{MT}_{\text {sat }}^{\mathrm{B} 1}$ maps with the T1-weighted MNI template image was achieved using the Auto-Reorient function (hMRI toolbox) and this was applied on the NODDI maps as well. Second, both $\mathrm{MT}_{\text {sat }}^{\mathrm{B} 1}$ maps of each subject (corresponding to two sessions) were registered to the mid-point average using the Pairwise Longitudinal Registration (SPM12). Hereby, values below zero and above 10 were excluded to improve the registration. Third, the resulting mid-point average image was normalized to the MNI space using the DARTEL-based (Ashburner, 2007) Spatial Processing module (hMRI toolbox). Fourth, a combined deformation field was generated per subject and session, combining the deformation fields from steps 2 and 3.

\section{Computation of $\mathbf{M V F}_{\mathrm{MR}}, \mathrm{AVF}_{\mathrm{MR}}$ and $\mathrm{g}_{\mathrm{MR}}$}

In this section, our approach to estimating MVF and AVF from the measured MR parameters is introduced. The MR-based MVF $\left(\mathrm{MVF}_{\mathrm{MR}}\right)$ was assumed to be proportional to $\mathrm{MT}_{\text {sat }}$ without intercept, following (Mohammadi and Callaghan, 2020):

$$
\mathrm{MVF}_{\mathrm{MR}}=\alpha \mathrm{MT}_{\mathrm{sat}}
$$

The proportionality constant $\alpha$ was estimated from Equation (2) in a region where the histological MVF $\left(\mathrm{MVF}_{\text {hist }}\right)$ was known. Due to the lack of own histological data, we used published histological data which contain the frequency distribution of inner-axon radius $(r)$ and myelin sheath thickness $(m)$ of 2,400 myelinated fibers in the medullary pyramids of a 71 years old human (see Table $\mathbf{1}$ in Graf von Keyserlingk and Schramm, 1984). The total volume (TV) of the sample is the sum of the total volume of myelinated axons $\left(\mathrm{TAV}_{\mathrm{m}}\right)$, unmyelinated axons $\left(\mathrm{TAV}_{\mathrm{u}}\right)$, myelin volume (TMV), and extracellular volume (TEV). TAV $\mathrm{m}$ was calculated as $\sum_{i=1}^{\mathrm{N}_{\mathrm{m}}} \pi \mathrm{r}_{i}^{2}$ with $i$ indexing the $\mathrm{N}_{\mathrm{m}}$ myelinated axons only, and TMV was computed as $\sum_{i=1}^{N_{\mathrm{m}}} \pi\left(\mathrm{r}_{i}+\mathrm{m}_{i}\right)^{2}-\mathrm{TAV}_{\mathrm{m}}$. $\mathrm{TAV}_{\mathrm{u}}$, while not reported in Graf von Keyserlingk and Schramm (1984), was found to be 


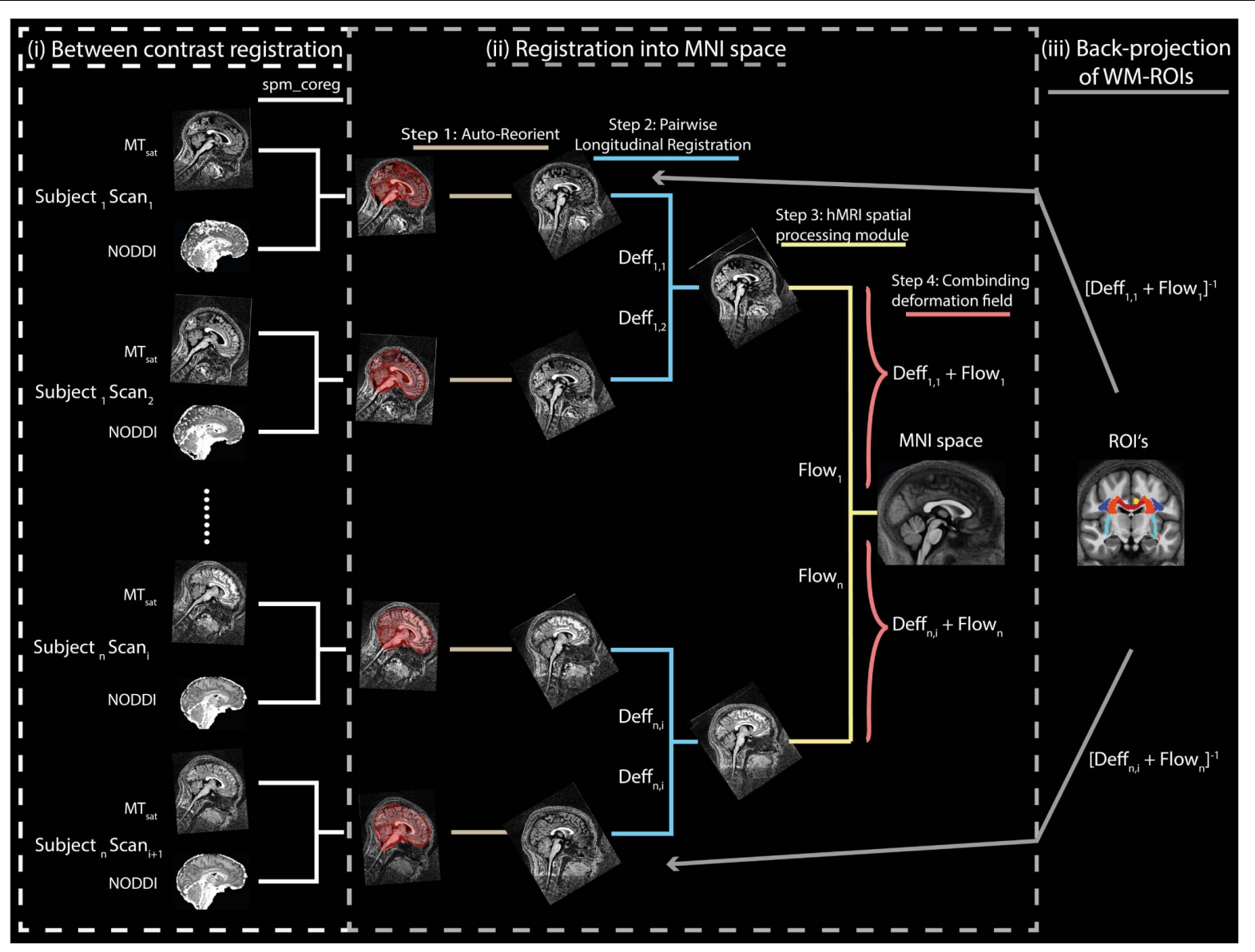

FIGURE 1 | Illustration of the spatial alignment pipeline of the $\mathrm{MT}_{\text {sat }}$ and NODDI maps. The pipeline consists of (i) co-registration between $\mathrm{MT}_{\text {sat }}$ and NODDI maps (driven by ODI map), (ii) normalization into MNI space, and (iii) back-projection of ROls into the native space. Note that each subject consists of two sets of images acquired in separate sessions. In the co-registration step (section "Co-registration"), the white matter (WM) tissue probability map (TPM) of the ODI was co-registered to the WM TMP of the MT sat in each subject and session using rigid-body registration (spm_coreg algorithm, SPM12). The resulting transformation was applied to all other NODDI maps as well. In the normalization step (section "Normalization"), $\mathrm{MT}_{\text {sat }}$ maps were roughly aligned with the T1-weighted MNI template in each subject and session using the Auto-Reorient function. The realigned $\mathrm{MT}_{\text {sat }}$ maps from both sessions were then registered to their mid-point average using the Pairwise Longitudinal Registration (SPM12). In each subject, the mid-point average MT sat map was normalized to the MNI space using the DARTEL-based (Ashburner, 2007) Spatial Processing module. Finally, all deformation fields were converted to a single deformation field and applied on the NODDI maps. In the last step (section "Region of Interest Selection"), the ROIs and the WM masks were back-projected into the native space using the inverse of the combined deformation field.

approximately $43 \%$ of $\mathrm{TAV}_{\mathrm{m}}$ for multiple mammals (Swadlow et al., 1980; LaMantia and Rakic, 1990; Olivares et al., 2001; Wang et al., 2008; Liewald et al., 2014). Note that the aforementioned papers typically reported the unmyelinated axons as $30 \%$ of the total volume of axons, which corresponds to $43 \%$ ( $=\frac{0.3}{1-0.3}$. 100) of $\mathrm{TAV}_{\mathrm{m}}$. EVF was estimated to be $25 \%$, according to Lehmenkühler et al. (1993), Nicholson and Hrabìtová (2017), Tønnesen et al. (2018). Finally, MVF was calculated as

$$
\mathrm{MVF}_{\text {hist }} \approx \frac{1}{\mathrm{TV}} \sum_{j=1}^{\mathrm{N}} \pi\left(\left(\mathrm{r}_{j}+\mathrm{m}_{j}\right)^{2}-\mathrm{r}_{j}^{2}\right)
$$

with $\mathrm{j}$ indexing all $\mathrm{N}$ fibers, yielding $\mathrm{MVF}_{\text {hist }} \approx 0.3623$. Plugging this value into Equation (2) (assuming that $\mathrm{MVF}_{\mathrm{MR}} \approx \mathrm{MVF}_{\text {hist }}$ ) along with the group-average $\mathrm{MT}_{\text {sat }}$ within the medullary pyramids (see Figure 2 for ROI definition) yielded an $\alpha$ of 0.2496 for $\mathrm{MT}_{\text {sat }}^{\mathrm{B} 1}, 0.2414$ for $\mathrm{MT}_{\text {sat }}^{\mathrm{UN}}$, and 0.2884 for $\mathrm{MT}_{\text {sat }}^{\mathrm{NO}}$.

The MR-based AVF $\left(\mathrm{AVF}_{\mathrm{MR}}=\left(1-\mathrm{MVF}_{\mathrm{MR}}\right) \mathrm{AWF}_{\mathrm{MR}}\right)$ was calculated as

$$
\mathrm{AVF}_{\mathrm{MR}}=\left(1-\alpha \mathrm{MT}_{\mathrm{sat}}\right)\left(1-v_{\mathrm{iso}}\right) \nu_{\mathrm{icvf}}
$$

where $\mathrm{AWF}=\left(1-v_{\text {iso }}\right) \nu_{\text {icvf }}$ is the axonal water fraction estimated from the NODDI parameters (Stikov et al., 2015) and $\mathrm{MVF}_{\mathrm{MR}}=\alpha \mathrm{MT}_{\text {sat }}$. The MR g-ratio was then computed according to Stikov et al. $(2011,2015)$

$$
\mathrm{g}_{\mathrm{MR}}=\sqrt{1-\frac{\mathrm{MVF}_{\mathrm{MR}}}{\mathrm{MVF}_{\mathrm{MR}}+\mathrm{AVF}_{\mathrm{MR}}}}
$$

Note that three versions of $\mathrm{MT}_{\text {sat }}, \mathrm{AVF}_{\mathrm{MR}}$, and $\mathrm{g}_{\mathrm{MR}}$ were generated according to notation in section "Data Processing": (i) 
TABLE 1 | Group-averaged mean and standard deviation (SD) of $g_{M R}^{B 1}$, MVF ${ }_{M R}^{B 1}$, and AVF $F_{M R}^{B 1}$ in 21 high-SNR ROls.

\begin{tabular}{|c|c|c|c|c|}
\hline Name & Acronym & $\mathrm{g}_{\mathrm{MR}}^{\mathrm{B1}}$ mean $\pm \mathrm{SD}$ & $\mathrm{AVF}_{\mathrm{MR}}^{\mathrm{B} 1}$ mean $\pm \mathrm{SD}$ & $\mathrm{MVF}_{\mathrm{MR}}^{\mathrm{B} 1}$ mean $\pm \mathrm{SD}$ \\
\hline Anterior limb of internal capsule right & ACL $r$ & $0.688 \pm 0.029$ & $0.384 \pm 0.052$ & $0.419 \pm 0.022$ \\
\hline Retrolenticular part of internal capsule left & $\mathrm{RICl}$ & $0.665 \pm 0.020$ & $0.341 \pm 0.025$ & $0.428 \pm 0.023$ \\
\hline Anterior corona radiata right & ACR $r$ & $0.651 \pm 0.012$ & $0.321 \pm 0.014$ & $0.435 \pm 0.014$ \\
\hline Anterior corona radiata left & ACR I & $0.644 \pm 0.015$ & $0.313 \pm 0.014$ & $0.440 \pm 0.018$ \\
\hline Superior corona radiata right & SCR $r$ & $0.679 \pm 0.014$ & $0.356 \pm 0.018$ & $0.413 \pm 0.087$ \\
\hline Superior corona radiata left & SCR I & $0.674 \pm 0.013$ & $0.350 \pm 0.016$ & $0.419 \pm 0.017$ \\
\hline Genu of corpus callosum & GCC & $0.642 \pm 0.020$ & $0.315 \pm 0.021$ & $0.445 \pm 0.024$ \\
\hline Body of corpus callosum & $\mathrm{BCC}$ & $0.657 \pm 0.021$ & $0.328 \pm 0.025$ & $0.425 \pm 0.020$ \\
\hline Posterior corona radiata right & PCR $r$ & $0.662 \pm 0.019$ & $0.326 \pm 0.025$ & $0.416 \pm 0.019$ \\
\hline Posterior corona radiata left & PCR I & $0.667 \pm 0.018$ & $0.337 \pm 0.023$ & $0.418 \pm 0.019$ \\
\hline Posterior thalamic radiation right & PTR $r$ & $0.643 \pm 0.016$ & $0.308 \pm 0.017$ & $0.438 \pm 0.018$ \\
\hline Posterior thalamic radiation left & PTR I & $0.645 \pm 0.017$ & $0.313 \pm 0.016$ & $0.438 \pm 0.020$ \\
\hline Sagittal stratum left & SAS I & $0.645 \pm 0.021$ & $0.314 \pm 0.020$ & $0.439 \pm 0.025$ \\
\hline External capsule right & EXC $r$ & $0.683 \pm 0.020$ & $0.359 \pm 0.023$ & $0.410 \pm 0.028$ \\
\hline External capsule left & EXCI & $0.682 \pm 0.025$ & $0.357 \pm 0.023$ & $0.408 \pm 0.034$ \\
\hline Cingulum left & CGM I & $0.661 \pm 0.023$ & $0.330 \pm 0.028$ & $0.422 \pm 0.029$ \\
\hline Fornix/Stria terminalis left & FNXI & $0.669 \pm 0.027$ & $0.349 \pm 0.036$ & $0.426 \pm 0.028$ \\
\hline Superior longitudinal fasciculus right & SLFr & $0.666 \pm 0.016$ & $0.334 \pm 0.017$ & $0.418 \pm 0.022$ \\
\hline Superior longitudinal fasciculus left & SLF I & $0.668 \pm 0.013$ & $0.340 \pm 0.015$ & $0.420 \pm 0.020$ \\
\hline Superior fronto-occipital fasciculus right & SFO $r$ & $0.678 \pm 0.020$ & $0.361 \pm 0.031$ & $0.422 \pm 0.020$ \\
\hline Superior fronto-occipital fasciculus left & SFO I & $0.672 \pm 0.021$ & $0.350 \pm 0.029$ & $0.424 \pm 0.020$ \\
\hline
\end{tabular}

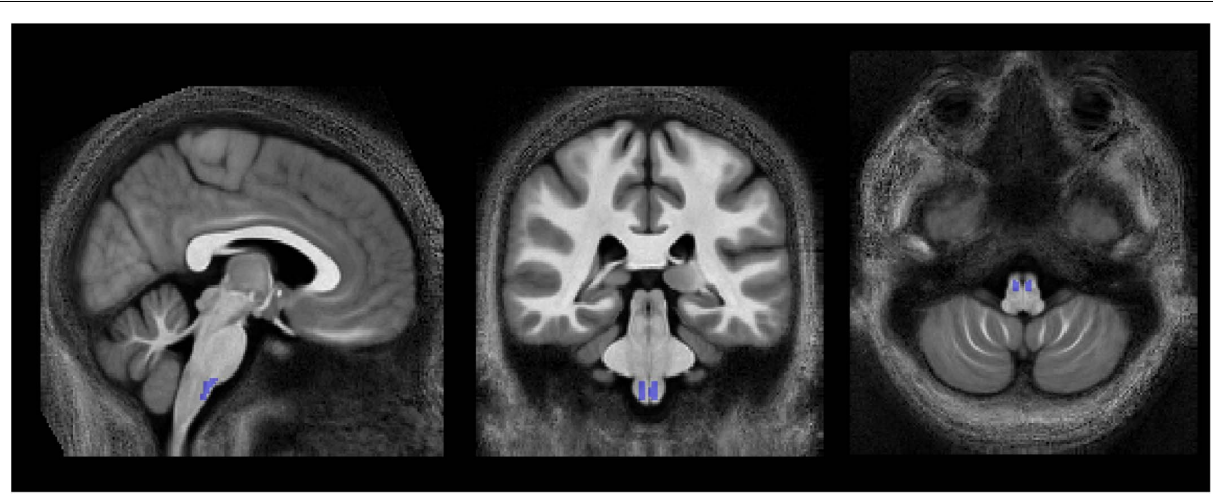

FIGURE 2 | Location of the pyramidal tracts in the medulla oblongata ROI, overlaid on the group-averaged $\mathrm{MT}_{\text {sat }}^{\mathrm{B} 1}$ map, that was used to determine the calibration constant, converting MT sat into MVF $F_{M R}$ (section "Computation of MVF $F_{M R}$, AVF $F_{M R}$, and gMR"). To create this ROI, the corticospinal tract ROI of the JHU-ICBM-DTI-81 atlas, which extends across the pons and medulla pyramids, was modified to cover only the medulla pyramids. Left-right position: $\mathrm{X}=82$; anterior-posterior position: $Y=77$; superior-inferior position, $Z=30$.

$\mathrm{MVF}_{\mathrm{MR}}^{\mathrm{NO}}, \mathrm{AVF}_{\mathrm{MR}}^{\mathrm{NO}}, \mathrm{g}_{\mathrm{MR}}^{\mathrm{NO}}$ for no correction, (ii) $\mathrm{MVF}_{\mathrm{MR}}^{\mathrm{B} 1}, \mathrm{AVF}_{\mathrm{MR}}^{\mathrm{B} 1}$, and $\mathrm{g}_{\mathrm{MR}}^{\mathrm{Bl}}$ for $\mathrm{B}_{1}+$ reference measurement, and (iii) $\mathrm{MVF}_{\mathrm{MR}}^{\mathrm{UN}}$, $\mathrm{AVF}_{\mathrm{MR}}^{\mathrm{UN}}$, and $\mathrm{g}_{\mathrm{MR}}^{\mathrm{UN}}$ for UNICORT $\mathrm{B}_{1}+$ correction.

\section{Definition of White Matter Masks}

As $\mathrm{g}_{\mathrm{MR}}$ and its constituents $\left(\mathrm{MVF}_{\mathrm{MR}}, \mathrm{AVF}_{\mathrm{MR}}\right)$ are defined only in the WM, we restricted the analysis to the WM by creating binary WM masks (Mohammadi and Callaghan, 2020). WM tissue probability maps (WM-TPM) were created for each subject by segmenting $\mathrm{AWF}$ and $\mathrm{MT}_{\text {sat }}^{\mathrm{B} 1}$ using the hMRI toolbox, and taking their intersection according to Mohammadi and Callaghan (2020). In two subjects, the $\mathrm{MT}_{\text {sat }}^{\mathrm{B} 1}$ segmentation was of insufficient quality for segmentation and was replaced by the $\mathrm{R}_{1}^{\mathrm{B}_{1}}$ map. A groupspecific binary WM mask $\left(\mathrm{WM}_{\text {group }}\right)$ was generated by averaging all individual WM-TPMs in the MNI space and thresholding it at 0.95 .

A so-called high-SNR WM $\mathrm{Wroup}_{\text {was }}$ also defined by taking the intersection of the $\mathrm{WM}_{\text {group }}$ and a binary signal-to-noise ratio (SNR) map. Hereby, the latter was used to reduce the number of voxels with unrealistically high values of $\nu_{\text {icvf }}\left(\nu_{\text {icvf }} \geq 0.999\right)$. In 6 of 25 subjects, an SNR map was created by dividing the mean $b_{0}$ image by a single noise estimate in the native space and multiplied by the square root of the number of $b_{0}$ images per DWI dataset $(n=12)$. The noise was estimated within a noise ROI outside the brain in 72 images ( 6 subjects, both timepoints and 6 b0 images 

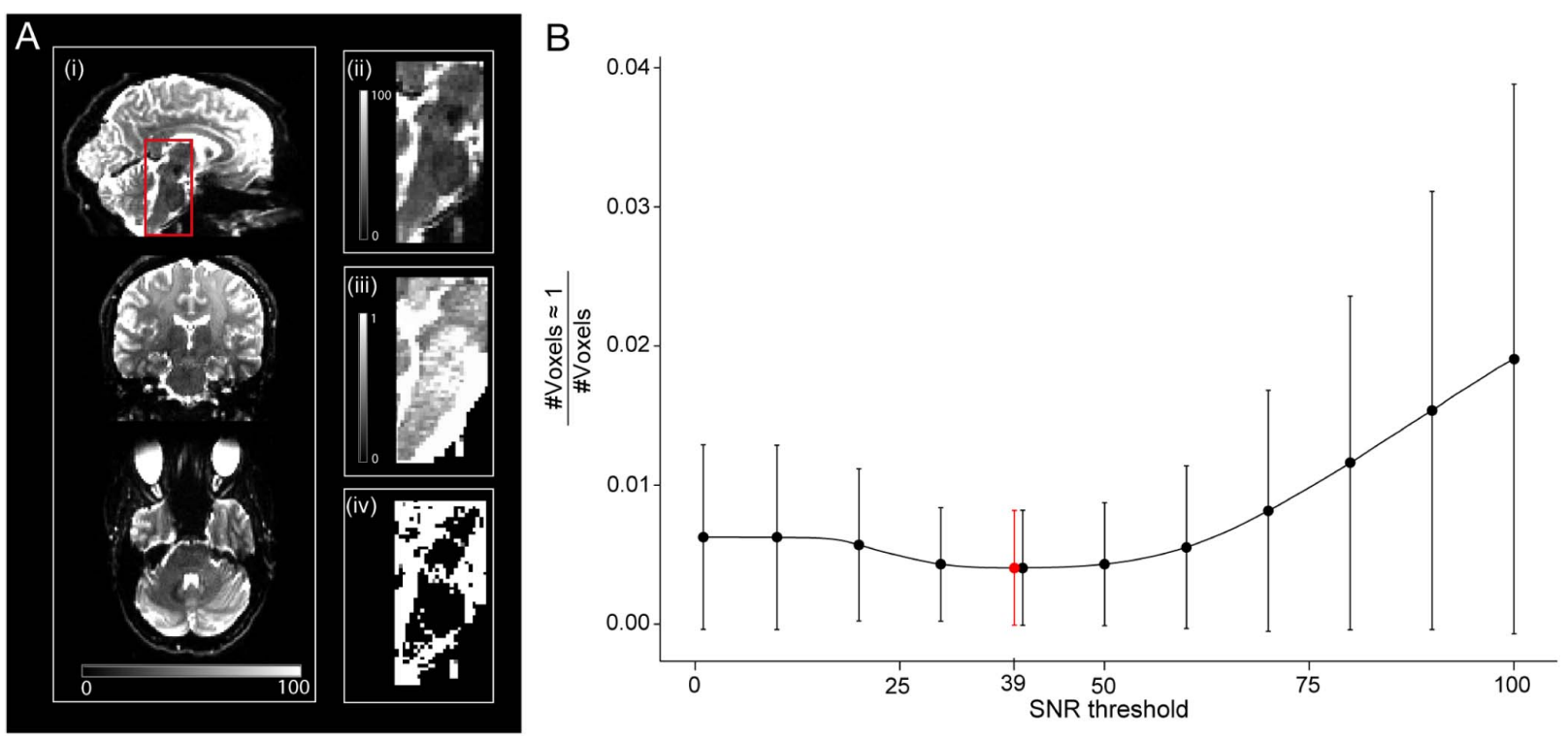

FIGURE 3 | Relationship between signal-to-noise ratio (SNR) and unrealistically high $v_{\text {icvf }}$ values - here defined as $v_{\text {icvf }} \geq 0.999$. (A) Sagittal, coronal, and axial view of the whole-brain SNR map (i), with a zoom-in view of the brainstem (ii). The brainstem is characterized by low SNR due to the spatial characteristics of the receive coil array (ii) and high occurrence of unrealistically high vicvf (iii), also shown as a binary mask (iv). (B) Given the co-occurrence of low SNR and unrealistically high $v_{\text {icvf }}$, a binary SNR mask was created to exclude low-SNR voxels. To determine the optimal threshold for the SNR mask, the ratio between the number of voxels with unrealistically high $v_{\text {icvf }}$ and the total number of voxels within the mask were plotted against the SNR threshold. The solid dots and error bars represent the group mean and group standard deviation of the ratio, respectively. The SNR value that yielded the minimum of this ratio was considered optimal (SNR $=39$, shown in red).

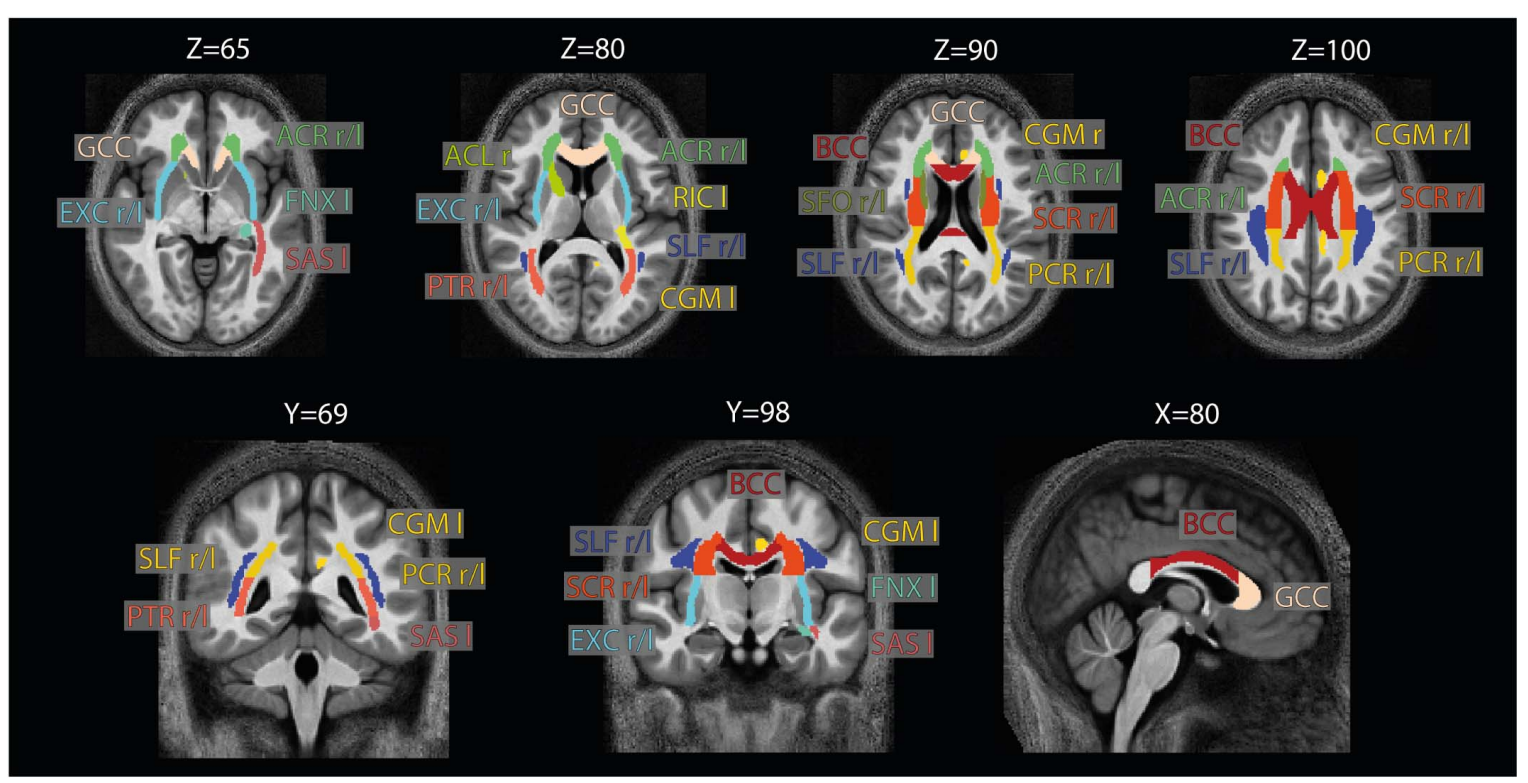

FIGURE 4 | Location of the ROls used for analysis. The 21 high-SNR ROls (listed in Table 1) are part of the JHU-ICBM-DTI-81 WM atlas (Hua et al., 2008) and are displayed here on the group-averaged normalized $\mathrm{MT}_{\text {sat }}^{\mathrm{B} 1}$ image. Note that for $\mathrm{ROI}$ analysis, the ROls were projected into the native space using the inverse of the combined deformation field.

each) using the ACID toolbox, with the values averaged to obtain a single noise estimate. The threshold for SNR maps to create binary SNR map was chosen such that it minimizes the ratio between the number of artifactual voxels where $\nu_{\text {icvf }} \geq 0.999$ and the total number of voxels in the SNR mask (Figure 3B), yielding a value of 39. This was motivated by the observation that unrealistically high $v_{\text {icvf }}$ values typically occur in low-SNR areas (Figures 3Aii,iii). This threshold selection represents a tradeoff between removing unrealistic voxels while retaining as many voxels as possible. 
TABLE 2 | Summary statistics of $g_{M R}^{B 1}, A V F_{M R}^{B 1}$, and MVF $F_{M R}^{B 1}$.

\begin{tabular}{lccccc}
\hline & $\boldsymbol{\Delta}_{\mathbf{D R}}$ & $\mathbf{m i n}_{\mathbf{i} \in \mathbf{R O I}}$ & $\max _{\mathbf{i} \in \mathbf{R O I}}$ & mean & SD \\
\hline $\mathrm{g}_{\mathrm{MR}}^{\mathrm{B} 1}$, & 0.046 & 0.642 & 0.688 & 0.664 & 0.014 \\
AVF $_{\mathrm{MR}}^{\mathrm{B} 1}$ & 0.076 & 0.308 & 0.384 & 0.337 & 0.020 \\
MVF $_{\mathrm{MR}}^{\mathrm{B} 1}$ & 0.037 & 0.408 & 0.445 & 0.425 & 0.010 \\
\hline
\end{tabular}

This table lists the dynamic range $\left(\triangle_{D R}\right)$, lowest ( $\left.\min _{i \in R O I}\right)$ and highest ( max $_{i \in R O I}$ ) $R O I$ average value, mean value of the 21 analyzed ROl's (mean) with its corresponding standard deviation (SD).

\section{Region of Interest Selection}

For the region of interest (ROI) analysis, the JHU-ICBM-DTI$81 \mathrm{WM}$ atlas (Hua et al., 2008) was transformed into the native space using the inverse of the combined deformation field. Two sets of ROIs were defined: (i) whole-WM ROIs and (ii) highSNR ROIs, used for the main analysis. The whole-WM ROIs included those of the JHU-ICBM-DTI-81 WM atlas that were completely in $\mathrm{WM}_{\text {group }}$ defined in 2.6, yielding 43 ROIs (out of 48 , leaving out the column and body of the fornix, the left and right cingulum part in the vicinity to the hippocampus, and the left and right uncinate fasciculus). The high-SNR ROIs included only those whole-WM ROIs that overlapped with the high-SNR $\mathrm{WM}_{\text {group }}$ to at least 95\%, yielding 21 ROIs (Figure 4 and Table 2). For the analyses, group-averaged $\mathrm{g}_{\mathrm{MR}}, \mathrm{AVF}_{\mathrm{MR}}$, and $\mathrm{MVF}_{\mathrm{MR}}$ were calculated within the $\mathrm{WM}_{\text {group }}$. Note that averaging included both sessions of each subject for all analyses except for the analysis in section "Test-Retest Analysis of the Group-Averaged MR G-ratio, Axon, and Myelin Volume Fraction."

\section{Test-Retest Analysis of the Group-Averaged MR G-ratio, Axon, and Myelin Volume Fraction}

The group-averaged $\mathrm{g}_{\mathrm{MR}}^{\mathrm{B} 1}$ of the first and second session were compared within the previously mentioned 21 highSNR ROIs using Bland-Altman plots (Bland and Altman, 1986). In the Bland-Altmann plots, the differences in $\mathrm{g}_{\mathrm{MR}}^{\mathrm{B} 1}$ between the first $\left(\mathrm{g}_{\mathrm{MR}}^{\mathrm{Bl}}\right)$ and second $\left(\mathrm{g}_{\mathrm{MR}_{2}}^{\mathrm{Bl}}\right)$ session $\left(\delta_{i}^{\text {retest }}=\left(\mathrm{g}_{\mathrm{MR}_{1}}^{\mathrm{Bl}}\right)_{i}-\left(\mathrm{g}_{\mathrm{MR}_{2}}^{\mathrm{Bl}}\right)_{i}\right)$ were plotted against their means $\left(\operatorname{mean}_{i}^{\text {retest }}=\frac{\left.\left(\mathrm{g}_{\mathrm{MR}}\right)_{i}+\left(\mathrm{g}_{\mathrm{MR} 2}^{\mathrm{Bl}}\right)_{i}\right)}{2}\right)$, where $i$ is the index of ROI $i$. Bias captures the offset $\left(\bar{\delta}^{\text {retest }}=\frac{1}{21} \sum_{i=1}^{21} \delta_{i}^{\text {retest }}\right)$, while error $\left(\epsilon^{\text {retest }}=1.96 \cdot \sqrt{\frac{1}{20} \sum_{i=1}^{21}\left(\delta_{i}^{\text {retest }}-\bar{\delta}^{\text {retest }}\right)}\right)$ captures the variation between the first and second scan within the $i^{\text {th }}$ ROI. The computed $\bar{\delta}^{\text {retest }}$ and $\epsilon^{\text {retest }}$ were normalized by the dynamic range $\left(\triangle_{\mathrm{DR}}\right)$ of $\mathrm{g}_{\mathrm{MR}}^{\mathrm{B} 1}$ within the high-SNR ROIs, defined as $\quad \triangle_{\mathrm{DR}}=\max _{\mathrm{i} \in \mathrm{ROI}}\left(\mathrm{mean}_{\mathrm{i}}^{\text {retest }}\right)-\min _{\mathrm{i} \in \mathrm{ROI}}\left(\right.$ mean $\left._{\mathrm{i}}^{\text {retest }}\right)$, yielding the relative error $\left(\delta_{\mathrm{DR} \%}^{\text {retest }}=\frac{\epsilon^{\text {retest }}}{\Delta_{\mathrm{DR}}} \cdot 100\right)$ and relative bias $\left(\bar{\delta}_{\mathrm{DR} \%}^{\text {retest }}=\frac{\bar{\delta}^{\text {retest }}}{\Delta_{\mathrm{DR}}} \cdot 100\right)$. The same procedure was also applied to $\mathrm{AVF}_{\mathrm{MR}}^{\mathrm{B} 1}$ and $\mathrm{MVF}_{\mathrm{MR}}^{\mathrm{B} 1}$.

The distinction between bias and error is important, because while a potential bias can be retrospectively corrected, the error in the MR g-ratio method defines its sensitivity to detect differences between individuals, groups, or time points. To reliably capture these differences, the error must be significantly lower than the expected effect size.

\section{Influence of $B_{1}+$ Correction in the Group-Averaged MR G-ratio, Axon, and Myelin Volume Fraction}

Bland-Altman analysis was used to compare $g_{M R}$ with and without $\mathrm{B}_{1}+$ correction. In particular, the difference $\delta_{i}^{\mathrm{B1}}$ in $\mathrm{g}_{\mathrm{MR}}$ between $\left(\mathrm{g}_{\mathrm{MR}}^{\mathrm{B1}}\right)_{i}$, when using the reference method $\mathrm{B}_{1}+$ correction, and $\left(\mathrm{g}_{\mathrm{MR}}^{\mathrm{k}}\right)_{i}$, when using no $(\mathrm{k}=\mathrm{NO})$ or UNICORT $(\mathrm{k}=\mathrm{UN}) \mathrm{B}_{1}+$ correction: $\delta_{i}^{\mathrm{B} 1}=\left(\mathrm{g}_{\mathrm{MR}}^{\mathrm{B} 1}\right)_{i}-\left(\mathrm{g}_{\mathrm{MR}}^{\mathrm{k}}\right)_{i}$ was plotted against their mean: mean $_{i}^{\mathrm{B} 1}=\frac{\left(\mathrm{g}_{\mathrm{MR}}^{\mathrm{B} 1}\right)_{i}+\left(\mathrm{g}_{\mathrm{MR}}\right)_{i}}{2}$, with $i$ being the index of the 21 high-SNR ROIs. The bias and error associated with the lack of (or UNICORT) $\mathrm{B}_{1}+$ correction are defined as $\bar{\delta}^{\mathrm{B} 1}=\frac{1}{21} \sum_{i=1}^{21} \delta_{i}^{\mathrm{B} 1}$ and $\epsilon^{\mathrm{B} 1}=1.96 \cdot \sqrt{\frac{1}{20} \sum_{i=1}^{21}\left(\delta_{i}^{\mathrm{B} 1}-\bar{\delta}^{\mathrm{B} 1}\right)}$, respectively.

The computed $\epsilon^{\mathrm{B} 1}$ and $\bar{\delta}^{\mathrm{B} 1}$ were normalized by the dynamic range of $\mathrm{g}_{\mathrm{MR}}^{\mathrm{B1}}$ within the high-SNR ROIs, yielding the relative error $\left(\epsilon_{\mathrm{DR} \%}^{\mathrm{B} 1}=\frac{\epsilon^{\mathrm{B} 1}}{\triangle_{\mathrm{DR}}} \cdot 100\right)$ and relative bias $\left(\bar{\delta}_{\mathrm{DR} \%}^{\mathrm{B} 1}=\frac{\bar{\delta}^{\mathrm{B} 1}}{\triangle_{\mathrm{DR}}} \cdot 100\right)$. The same procedure was also applied to $A V F_{M R}$ and $\mathrm{MVF}_{\mathrm{MR}}$, comparing them to their respective reference method and dynamic range. For $\mathrm{MVF}_{\mathrm{MR}}$, the Bland-Altman analysis was additionally done using the whole-WM ROIs instead of the highSNR ROIs (see section "Region of Interest Selection") to assess the influence of including low-SNR voxels in the analysis.
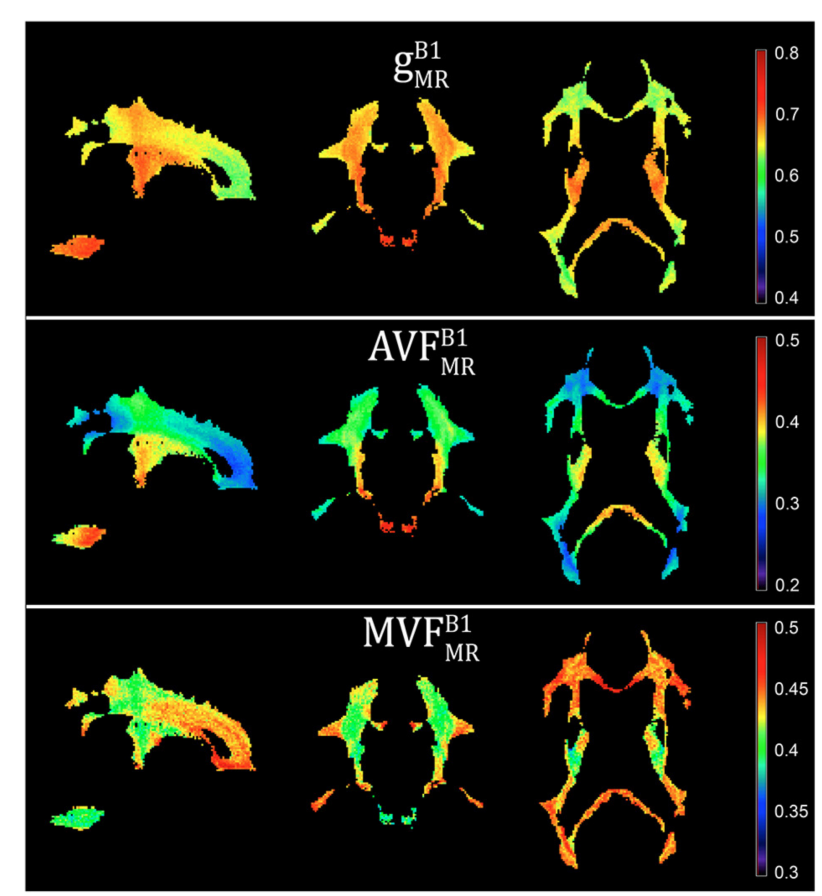

FIGURE 5 | Voxel-wise maps of group-averaged $g^{\mathrm{B} 1}{ }_{\mathrm{MR}}, \mathrm{AVF}^{\mathrm{B} 1} \mathrm{MR}$, and $M V F^{B 1}{ }_{M R}$, restricted to the group WM mask (cf. section "Definition of White Matter Masks"). Depicted are a single sagittal ( $x=100)$, coronal $(y=91)$, and axial $(z=85)$ slice. 


\section{Group Variability in MR G-ratio, Axon, and Myelin Volume Fraction}

To assess group variability for each correction method, the coefficient-of-variation (CoV) across subjects and sessions was calculated for $\mathrm{MVF}_{\mathrm{MR}}, \mathrm{AVF}_{\mathrm{MR}}$, and $\mathrm{g}_{\mathrm{MR}}$ in the MNI space after applying tissue-weighted smoothing (Tabelow et al., 2019), yielding: $\mathrm{CoV}_{M R}^{\mathrm{B1}}, \mathrm{CoV}_{\mathrm{MR}}^{\mathrm{UN}}$, and $\mathrm{CoV}_{\mathrm{MR}}^{\mathrm{NO}}$, where $\mathrm{MR} \in$ $\left\{g_{M R}, \mathrm{AVF}_{\mathrm{MR}}\right.$, and $\left.\mathrm{MVF}_{\mathrm{MR}}\right\}$. For tissue-weighted smoothing, a full width at half maximum Gaussian smoothing kernel of $6 \mathrm{~mm}$ was used. Bland-Altman analysis (see section "TestRetest Analysis of the Group-Averaged MR G-ratio, Axon, and Myelin Volume Fraction") was used to compare $\operatorname{CoV}_{\mathrm{MR}}^{\mathrm{UN}}$ and $\mathrm{CoV}_{\mathrm{MR}}^{\mathrm{NO}}$ against $\mathrm{CoV}_{\mathrm{MR}}^{\mathrm{B} 1}$ based on the reference method, yielding bias $\left(\bar{\delta}^{\mathrm{CoV}}\right)$ and error $\left(\epsilon^{\mathrm{CoV}}\right)$ values. A higher variability across the brain is expected to increase $\bar{\delta}^{\mathrm{CoV}}$ whereas a higher local variability is expected to increase $\epsilon \mathrm{CoV}$.

\section{RESULTS}

\section{G-ratio, Myelin, and Axonal Volume Fraction Across the White Matter}

Voxel-wise maps of group-averaged $\mathrm{g}_{\mathrm{MR}}^{\mathrm{B1}}, \mathrm{AVF}_{\mathrm{MR}}^{\mathrm{B1}}$, and $\mathrm{MVF}_{\mathrm{MR}}^{\mathrm{B1}}$ in WM are shown in Figure 5. The group-averaged mean and standard deviation of $\mathrm{g}_{\mathrm{MR}}^{\mathrm{B1}}, \mathrm{MVF}_{\mathrm{MR}}^{\mathrm{B1}}$, and $\mathrm{AVF}_{\mathrm{MR}}^{\mathrm{B}}$ in 21 highSNR ROIs are reported in Table $\mathbf{1}$ and Figure 6. The dynamic range $\left(\triangle_{\mathrm{DR}}\right)$, minimum and maximum values, and mean and standard deviation of $\mathrm{g}_{\mathrm{MR}}^{\mathrm{B} 1}, \mathrm{AVF}_{\mathrm{MR}}^{\mathrm{B} 1}$, and $\mathrm{MVF}_{\mathrm{MR}}^{\mathrm{B} 1}$ across ROIs are listed in Table 2. The largest $\mathrm{g}_{\mathrm{MR}}^{\mathrm{B} 1}$ and $\mathrm{AVF}_{\mathrm{MR}}^{\mathrm{B} 1}$ were found in the right anterior limb of the internal capsule (0.688 and 0.384 , respectively), while the largest $\mathrm{MVF}_{\mathrm{MR}}^{\mathrm{B1}}$ was in the genu of corpus callosum (0.445), where also the lowest $\mathrm{g}_{\mathrm{MR}}^{\mathrm{B} 1}(0.642)$ can be found. The lowest $\mathrm{AVF}_{\mathrm{MR}}^{\mathrm{B} 1}$, and $\mathrm{MVF}_{\mathrm{MR}}^{\mathrm{B1}}$ were found in the right posterior thalamic radiation $\left(\mathrm{AVF}_{\mathrm{MR}}^{\mathrm{BI}}=0.308\right)$ and in the left external capsule $\left(\mathrm{MVF}_{\mathrm{MR}}^{\mathrm{B1}}=0.408\right)$, respectively. The $\triangle_{\mathrm{DR}}$ was the smallest for $\mathrm{MVF}_{\mathrm{MR}}^{\mathrm{B1}}(0.037)$, followed by $\mathrm{g}_{\mathrm{MR}}^{\mathrm{B} 1}(0.046)$ and $\mathrm{AVF}_{\mathrm{MR}}^{\mathrm{B} 1}$ (0.076).

\section{Test-Retest Analysis of the Group-Averaged MR G-ratio, Axon, and Myelin Volume Fraction}

The relative error $\left(\epsilon_{\mathrm{DR} \%}^{\text {retest }}\right)$ and bias $\left(\bar{\delta}_{\mathrm{DR} \%}^{\text {retest }}\right)$ values of the test-retest analysis are summarized in Table 3 and shown as Bland-Altmann plots in Figure 7. The test-retest analysis

TABLE 3 | Bias and error between scans, in $g_{M R}^{B 1}, A V F_{M R}^{B 1}$, and $M V F_{M R}^{B 1}$.

\begin{tabular}{lcccc}
\hline MAP & $\bar{\delta}^{\text {retest }}$ & $\epsilon^{\text {retest }}$ & $\bar{\delta}_{\text {DR\% }}^{\text {retest }}$ & $\epsilon_{\text {DR\% }}^{\text {retest }}$ \\
\hline g $_{\text {MR }}^{\text {B1 }}$ & 0.0021 & 0.0102 & 4.57 & 22.17 \\
AVF $_{\text {MR }}^{B 1}$ & 0.0006 & 0.0156 & 0.79 & 20.53 \\
MVF $_{\text {MR }}^{B 1}$ & -0.0031 & 0.0076 & -8.38 & 20.54
\end{tabular}

List of the bias $\left(\bar{\delta}^{\text {retest }}\right)$ and error ( $\left.\epsilon^{\text {retest }}\right)$ values, defined as in Figure 7, along with their relative value with respect to the dynamic range $\Delta_{\mathrm{DR}}: \epsilon_{D R \%}^{\text {retest }}=\frac{\mathrm{\epsilon}^{\text {retest }}}{\Delta D R} \cdot 100$; $\bar{\delta}_{D R \%}^{\text {retest }}=\frac{\bar{\delta}^{\text {retest }}}{\Delta_{D R}} \cdot 100$.

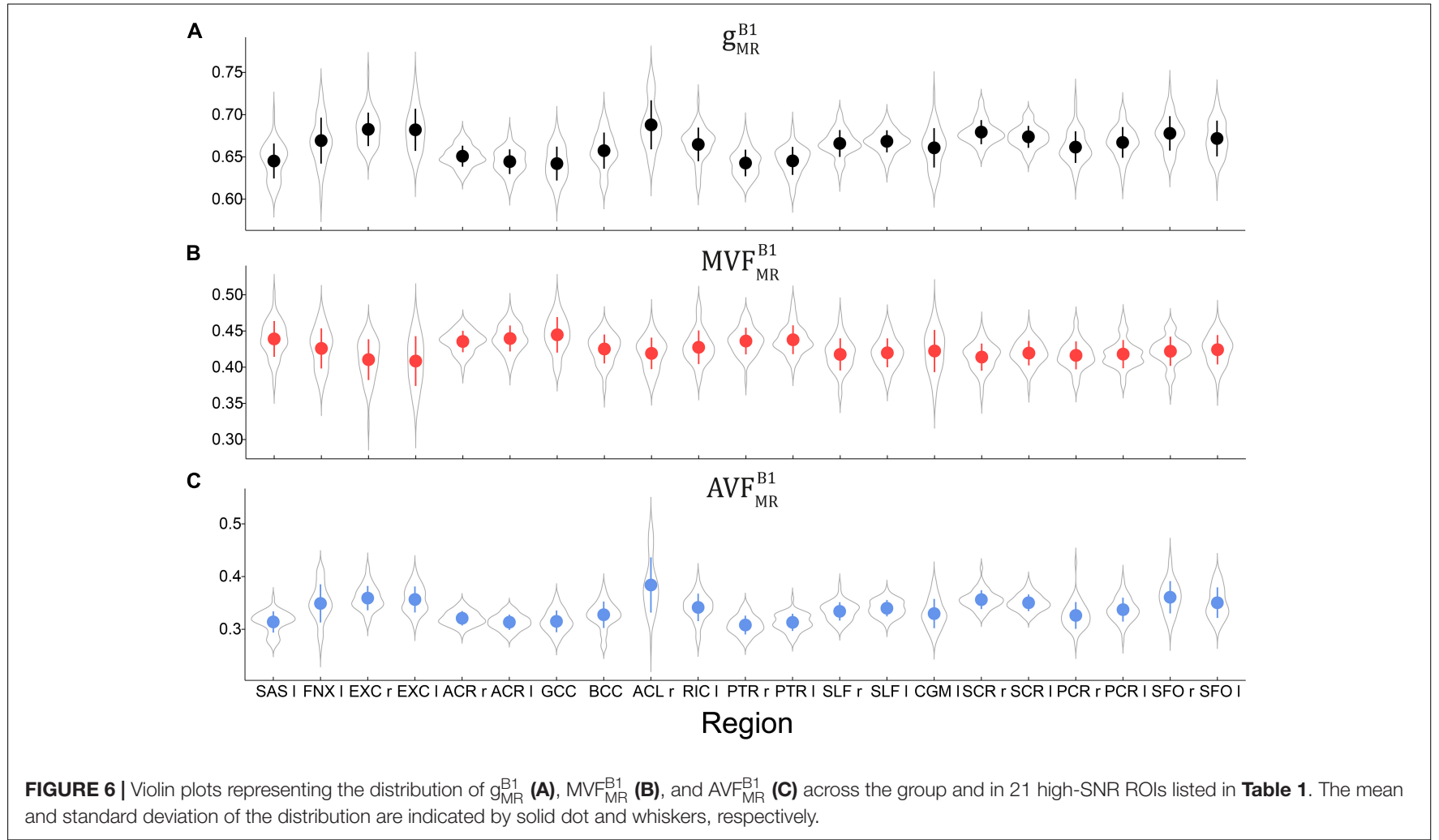




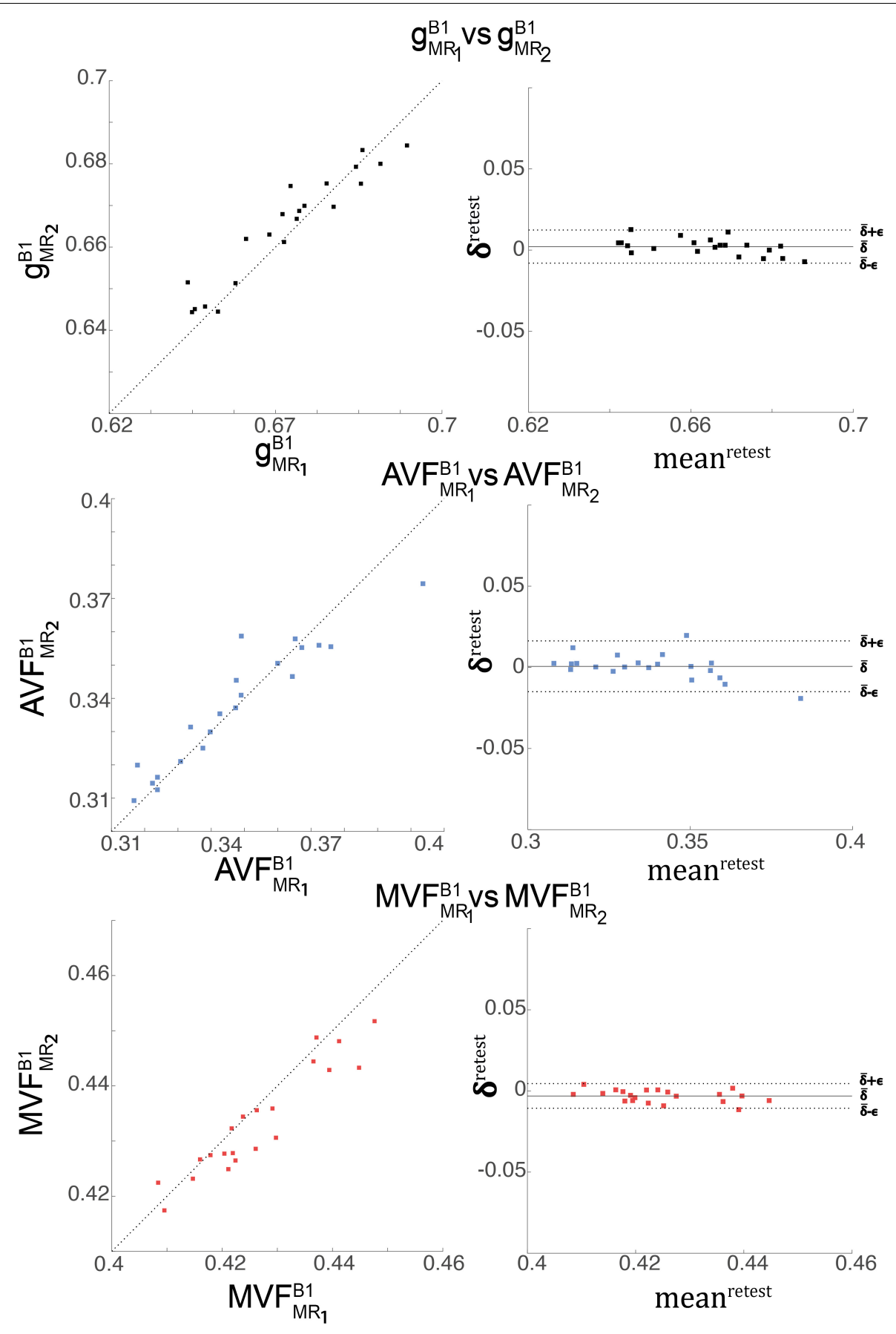

FIGURE 7 | Depicted are scatter and Bland-Altman plots of $g_{M R}^{B 1}$ (first row), $A V F_{M R}^{B 1}$ (second row), and $M V F_{M R}^{B 1}$ (third row) from two session across 21 WM regions (denoted high-SNR ROls, see Figure 4). The Bland-Altman plot illustrates the differences between values obtained from the two sessions (e.g., $g_{M R 1}^{B 1} v s . g_{M R 2}^{B 1}$; $\left.\delta_{i}^{\text {retest }}=\left(\mathrm{g}_{\mathrm{MR1}}^{\mathrm{B} 1}\right)_{i}-\left(\mathrm{g}_{\mathrm{MR2}}^{\mathrm{B} 1}\right)_{i}\right)$ against their mean (e.g., mean ${ }_{i}^{\text {retest }}=\frac{\left(\mathrm{g}_{\mathrm{MR1}}^{\mathrm{B} 1}\right)_{i}+\left(\mathrm{g}_{\mathrm{MR2} 2}^{\mathrm{B} 1}\right)_{i}}{2}$, with $i$ indexing the $\left.i^{\text {th }} \mathrm{ROI}\right)$. Each point in the scatter plot represents the group-averaged value in a single ROI. The bold black line represents the bias $\left(\delta^{\text {retest }}=\frac{1}{21} \sum_{i=1}^{21} \delta_{i}^{\text {retest }}\right)$, while the dashed line shows error $\left(\epsilon^{\text {retest }}=1.96 \cdot \operatorname{SD}\left(\delta_{i}^{\text {retest }}\right)\right)$ between the two sessions.

revealed a $\bar{\delta}_{\mathrm{DR} \%}^{\text {retest }}$ below an absolute value of $8.4 \%$ for each metric $\left(\mathrm{g}_{\mathrm{MR}}^{\mathrm{B} 1}, \mathrm{AVF}_{\mathrm{MR}}^{\mathrm{B1}}\right.$, and $\left.\mathrm{MVF}_{\mathrm{MR}}^{\mathrm{B1}}\right)$, where the $\mathrm{AVF}_{\mathrm{MR}}^{\mathrm{B} 1}$ showed the lowest $\bar{\delta}_{\mathrm{DR} \%}^{\text {retest }}$ with $0.79 \%$ (Figure 7 and Table 3).
The $\epsilon_{\mathrm{DR} \%}^{\text {retest }}$ was below $22.2 \%$ for each metric, where the $\mathrm{AVF}_{\mathrm{MR}}^{\mathrm{B} 1}$ showed the lowest $\epsilon_{\mathrm{DR} \%}^{\text {retest }}$ with $20.5 \%$ (Figure 7 and Table 3). 
TABLE 4 | Bias and error between methods, in $g_{M R}, A V F_{M R}$, and MVF $F_{M R}$.

\begin{tabular}{|c|c|c|c|c|}
\hline MAP & $\bar{\delta}^{\mathrm{B} 1}$ & $\epsilon^{B 1}$ & $\bar{\delta}_{\mathrm{DR} \%}^{\mathrm{B1}}$ & $\epsilon_{\mathrm{DR} \%}^{\mathrm{B} 1}$ \\
\hline$g_{M R}^{B 1}$ vs. $g_{M R}^{N O}$ & -0.041 & 0.017 & -89.13 & 36.96 \\
\hline$g_{M R}^{B 1}$ vs. $g_{M R}^{\cup N}$ & 0.014 & 0.005 & 30.44 & 10.87 \\
\hline $\mathrm{AVF}_{\mathrm{MR}}^{\mathrm{B} 1}$ Vs. $A V F_{\mathrm{MR}}^{\mathrm{NO}}$ & -0.031 & 0.012 & -40.79 & 15.79 \\
\hline $\mathrm{AVF}_{\mathrm{MR}}^{\mathrm{B} 1}$ vs. AVF $\mathrm{MR}$ & 0.011 & 0.004 & 14.47 & 5.26 \\
\hline $\mathrm{MVF}_{\mathrm{MR}}^{\mathrm{B} 1}$ vs. MVF $\mathrm{MR}_{\mathrm{MR}}^{\mathrm{NO}}$ & 0.053 & 0.022 & 143.24 & 59.46 \\
\hline $\mathrm{MVF}_{\mathrm{MR}}^{\mathrm{B} 1}$ vs. MVF $\mathrm{MR}$ & -0.018 & 0.006 & -48.65 & 16.22 \\
\hline EWM MVF ${ }_{M R}^{B 1}$ vs. MVF $F_{M R}^{N O}$ & 0.033 & 0.048 & 36.48 & 52.75 \\
\hline $\mathrm{EWM}_{\mathrm{MVF}}^{\mathrm{B} 1}$ vs. MVF $\mathrm{MR}$ & -0.012 & 0.022 & -13.08 & 23.96 \\
\hline
\end{tabular}

List of the bias $\left(\bar{\delta}^{B 1}\right)$ and error $\left(\epsilon^{B 1}\right)$ values as defined in Figure 9, along with their relative value with respect to the dynamic range $\triangle_{D R}: \epsilon_{D R \%}^{B 1}=\frac{\epsilon^{B 1}}{\triangle_{D R}} \cdot 100 ; \bar{\delta}_{D R \%}^{B 1}=$ $\frac{\bar{\delta}^{B 1}}{\triangle_{D R}} \cdot 100$. Note that the error and bias in the last two rows were obtained when using the whole-WM ROls instead of the high-SNR ROIs (see Supplementary Figure 1).

\section{Influence of $B_{1}+$ Correction on the Group-Averaged MR G-ratio, Axon, and Myelin Volume Fraction}

The relative error $\left(\epsilon_{\mathrm{DR} \%}^{\mathrm{B} 1}\right)$ and bias $\left(\bar{\delta}_{\mathrm{DR} \%}^{\mathrm{B} 1}\right)$ values of the $\mathrm{B}_{1}+$ correction analysis are summarized in Table $\mathbf{4}$ and shown as Bland-Altmann plots in Figures 8, 9. For g gR $_{\text {, compared to }}$ the no-correction case, UNICORT showed both lower $\epsilon_{\mathrm{DR} \%}^{\mathrm{B} 1}$
(UNICORT vs. no correction: $10.9 \%$ vs. $37.0 \%)$ and $\bar{\delta}_{\mathrm{DR} \%}^{\mathrm{B} 1}(30.4 \%$ vs. $-89.1 \%$ ). For both $\mathrm{AVF}_{\mathrm{MR}}$ and $\mathrm{MVF}_{\mathrm{MR}}$, UNICORT yielded lower $\epsilon_{\mathrm{DR} \%}^{\mathrm{B} 1}$ (UNICORT vs. no correction; $\mathrm{AVF}_{\mathrm{MR}}: 5.3 \%$ vs. $15.8 \% ; 16.2 \%$ vs. $59.5 \%)$ and lower $\bar{\delta}_{\mathrm{DR} \%}^{\mathrm{B} 1}\left(\mathrm{AVF}_{\mathrm{MR}}: 14.5 \%\right.$ vs.

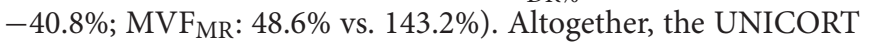
correction reduced the bias and error in the MR g-ratio and its constituents by roughly a factor of three. The lower $\epsilon_{\mathrm{DR} \%}^{\mathrm{B} 1}$ and $\bar{\delta}_{\mathrm{DR} \%}^{\mathrm{B} 1}$ associated with UNICORT was also reflected by the fact that values of $\mathrm{g}_{\mathrm{MR}}^{\mathrm{UN}}, \mathrm{AVF}_{\mathrm{MR}}^{\mathrm{UN}}$, and $\mathrm{MVF}_{\mathrm{MR}}^{\mathrm{UN}}$ (Figure 8, lower panel) lie closer to the unit slope line than values of $g_{M R}^{N O}, A_{M} F_{M R}^{N O}$, and $\mathrm{MVF}_{\mathrm{MR}}^{\mathrm{NO}}$ (Figure 8, upper panel). When computing $\epsilon_{\mathrm{DR} \%}^{\mathrm{B1}}$ and $\bar{\delta}_{\mathrm{DR} \%}^{\mathrm{B} 1}$ of $\mathrm{g}_{\mathrm{MR}}$ in the whole-WM ROIs (see Supplementary Figure 1), $\bar{\delta}_{\mathrm{DR}}^{\mathrm{B} 1}$ was consistently lower for both the no-correction case (whole-WM ROIs vs. high-SNR ROIs: $36.5 \%$ vs. $143.2 \%$ ) and UNICORT $(13.1 \%$ vs. $48.6 \%)$, whereas $\epsilon_{\mathrm{DR} \%}^{\mathrm{B} 1}$ was similar (no-correction: $52.8 \%$ vs. $59.5 \%$; UNICORT: $24.0 \%$ vs. $16.2 \%$ ).

\section{Group Variability in MR G-ratio, Axon, and Myelin Volume Fraction}

$\mathrm{g}_{\mathrm{MR}}$ showed on average smaller $\mathrm{CoV}$ than $\mathrm{AVF}_{\mathrm{MR}}$ and $\mathrm{MVF}_{\mathrm{MR}}$ (Figure 10). In all maps, the $\mathrm{CoV}$ was the highest in the deep brain areas. The relative error $\left(\frac{\epsilon^{\mathrm{CoV}}}{\mathrm{CoV}^{\mathrm{B1}}} \cdot 100\right)$ and bias $\left(\frac{\bar{\delta}^{\mathrm{CoV}}}{\mathrm{CoV}^{\mathrm{B1}}} \cdot 100\right)$ values of $\mathrm{CoV}$ with respect to the $\mathrm{B}_{1}+$ reference measurement
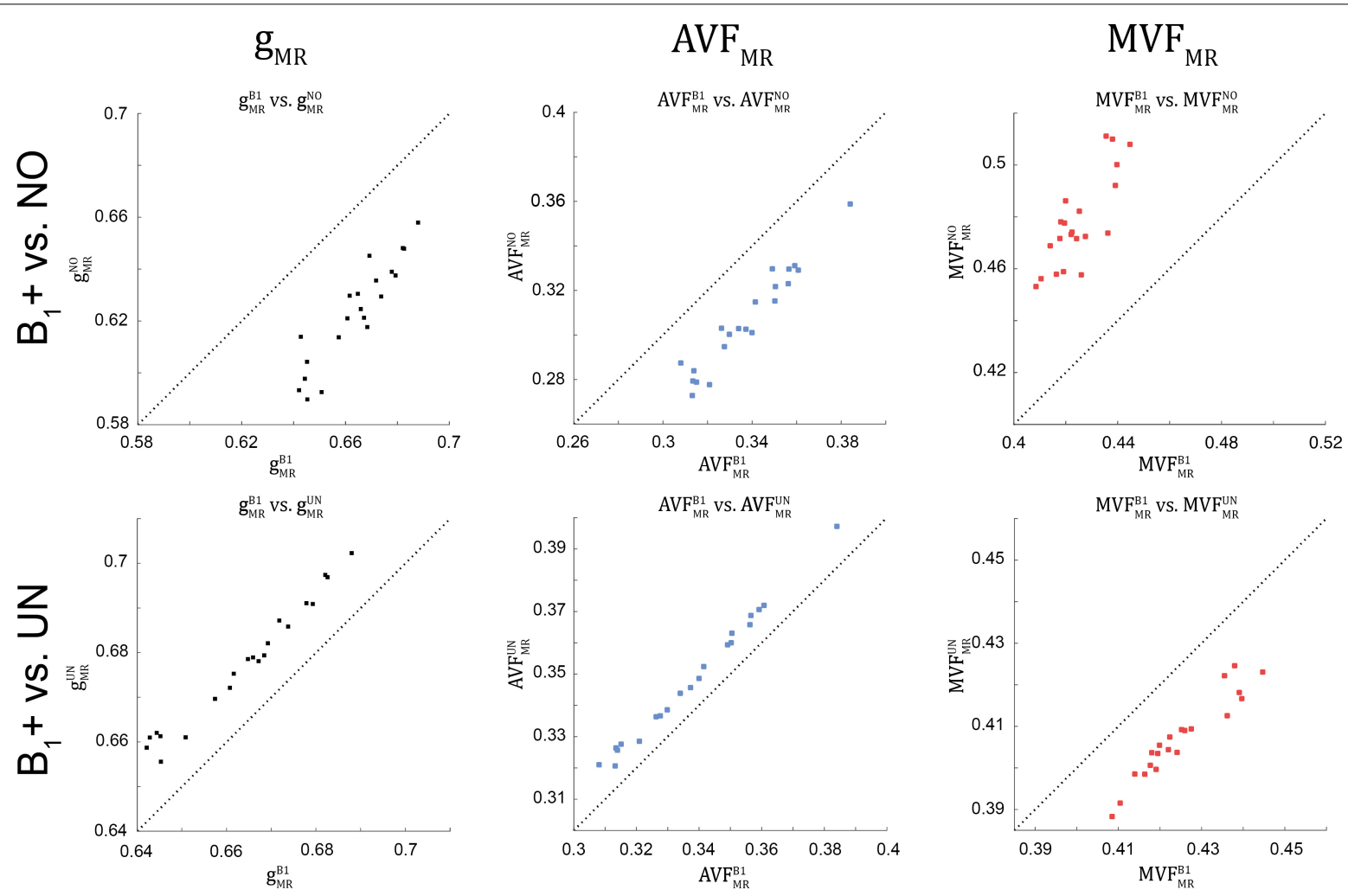

FIGURE 8 | Scatter plots of $\mathrm{gMR}_{\mathrm{M}}, \mathrm{AVF}_{\mathrm{MR}}$, and $\mathrm{MVF}_{\mathrm{MR}}$, plotting values obtained without $\mathrm{B}_{1}+$ correction (superscript: NO, top row) and with UNICORT $\mathrm{B}_{1}+$ correction (superscript: UN, bottom row) against values obtained with the reference method, i.e., $\mathrm{B}_{1}+$ field map correction (superscript: B1). A dashed unit slope line is plotted for reference. Each point in the scatter plot represents the group-averaged value in a single ROI (see Figure $\mathbf{4}$ for the locations of the 21 high-SNR ROIs). 

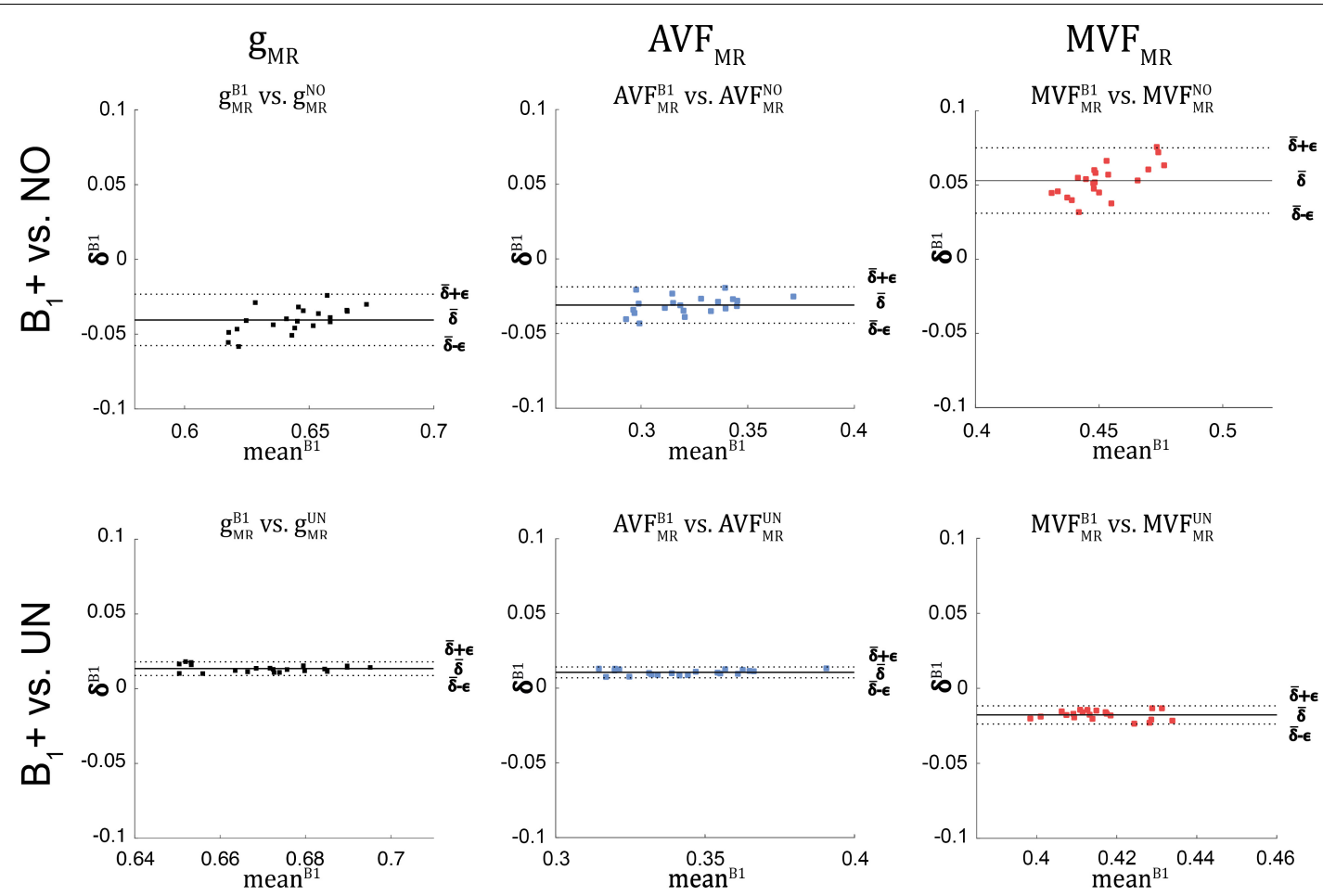

FIGURE 9 | Bland-Altman plots of $\mathrm{gMR}_{\mathrm{MR}}, \mathrm{AVF}_{\mathrm{MR}}$, and $\mathrm{MVF}_{\mathrm{MR}}$, comparing values obtained without $\mathrm{B}_{1}+$ correction (NO, top row) and with $\mathrm{UNICORT} \mathrm{B}_{1}+$ correction (UN, bottom row) against values obtained by $\mathrm{B}_{1}+$ field map correction (superscript: B1). The Bland-Altman plot illustrates the differences between values obtained by two different methods (reference vs. tested method); e.g., $\delta_{i}^{\mathrm{B} 1}=\left(\mathrm{g}_{\mathrm{MR}}^{\mathrm{B} 1}\right)_{i}-\left(\mathrm{g}_{\mathrm{MR}}^{\mathrm{k}}\right)_{i}$ against their mean $\left(\right.$ mean $_{i}^{\mathrm{B} 1}=\frac{\left(\mathrm{g}_{\mathrm{MR}}^{\mathrm{B}}\right)_{i}+\left(\mathrm{g}_{\mathrm{MR}}^{\mathrm{k}}\right)_{i}}{2}$, with $\mathrm{k}=\{\mathrm{UN}, \mathrm{NO}\}$ and $i$ indexing the $i^{\text {th }}$ $\mathrm{ROI}$ ). Each point in the scatter plot represents the group-averaged value in a single ROI (see Figure $\mathbf{4}$ for the locations of the 21 high-SNR ROIs). The bold black line represents the bias $\left(\bar{\delta}^{\mathrm{B} 1}=\sum_{i=1}^{21} \delta_{i}^{\mathrm{B1}}\right)$, while the dashed line shows error $\left(\epsilon^{\mathrm{B} 1}=1.96 \cdot \mathrm{SD}\left(\delta_{i}^{\mathrm{B} 1}\right)\right)$ between the reference and the tested method. Error and bias values averaged across all ROls and subjects are listed in Table $\mathbf{5}$.

are summarized in Table 5 and the error and bias are also displayed as Bland-Altman density plot in Figure 11. For g $g_{M R}$, compared to the no correction case, UNICORT showed similar $\epsilon^{\mathrm{CoV}}$ (UNICORT vs. no correction: $0.6 \%$ vs. $0.6 \%$ ) but lower $\bar{\delta} \mathrm{CoV}$ $(-0.1 \%$ vs. $-0.4 \%)$. UNICORT yielded higher $\epsilon^{\mathrm{CoV}}$ (UNICORT vs. no correction; $1.0 \%$ vs. $0.8 \%)$ and lower $\bar{\delta}^{\mathrm{CoV}}(-0.2 \%$ vs. $-0.4 \%)$ for $\mathrm{AVF}_{\mathrm{MR}}$, and higher $\epsilon^{\mathrm{CoV}}$ (1.2\% vs. $\left.0.4 \%\right)$ and higher $\bar{\delta}^{\mathrm{CoV}}(-0.5 \%$ vs. $-0.1 \%)$ for $\mathrm{MVF}_{\mathrm{MR}}$. The lower $\bar{\delta}^{\mathrm{CoV}}$ of $\mathrm{g}_{\mathrm{MR}}$ and $\mathrm{AVF}_{\mathrm{MR}}$ associated with UNICORT reveals itself as a slight shift of the points toward the unit slope line in the scatter density plot (Figure 12).

\section{DISCUSSION}

In this study, we showed that omitting the correction of the magnetization transfer saturation map $\left(\mathrm{MT}_{\mathrm{sat}}\right)$ for residual $\mathrm{B}_{1}+$ effects introduces large error and bias in the MR g-ratio and the constituents (myelin and axon volume fractions, or in short $\mathrm{MVF}_{\mathrm{MR}}$ and $\mathrm{AVF}_{\mathrm{MR}}$ ). We also demonstrated that this error and bias can be reduced by roughly a factor of three using the data-driven UNICORT $\mathrm{B}_{1}+$ correction (implemented in the hMRI toolbox, see text footnote 1 ) when a $B_{1}+$ field measurement is unavailable.

\section{The Effect of Omitting the $B_{1}+$ Field Measurement}

$\mathrm{MT}_{\text {sat }}$ have been often used as a proxy for the $\mathrm{MVF}_{\mathrm{MR}}$ in g-ratio weighted imaging (Mohammadi et al., 2015; Campbell et al., 2018; Ellerbrock and Mohammadi, 2018; Hori et al., 2018; Kamagata et al., 2019), because they are directly linked to the macromolecular pool with an intrinsic correction for underlying longitudinal relaxation time and $\mathrm{B}_{1}+$ field inhomogeneities effects (Helms et al., 2008). Despite the latter intrinsic correction for $\mathrm{B}_{1}+$ field inhomogeneities, we found that the residual $\mathrm{B}_{1}+$ effects on $\mathrm{MT}_{\text {sat }}$ map were still observable. In particular, the bias and error of the MR g-ratio $\left(g_{M R}\right)$ was about -89 and $37 \%$ higher, respectively, when omitting the $\mathrm{B}_{1}+$ correction. We found the same trend for $\mathrm{MVF}_{\mathrm{MR}}$ and $\mathrm{AVF}_{\mathrm{MR}}$; while the error and bias were even larger for $\mathrm{MVF}_{\mathrm{MR}}$ when $\mathrm{B}_{1}+$ correction was omitted, it was smaller but still substantial for the $\mathrm{AVF}_{\mathrm{MR}}$. We found that omitting $\mathrm{B}_{1}+$ leads to a substantially higher (more than 10-fold) bias in the MR g-ratio and its constituents when compared to a test-retest analysis of our data (Figure 7 and Table 3). Also, the error due to omitting the $\mathrm{B} 1+$ correction was twice as large as the error observed in the test retest analysis for the MR g-ratio and the MVF, whereas for AVF the errors were similar. We expect that the high error will be of particular 

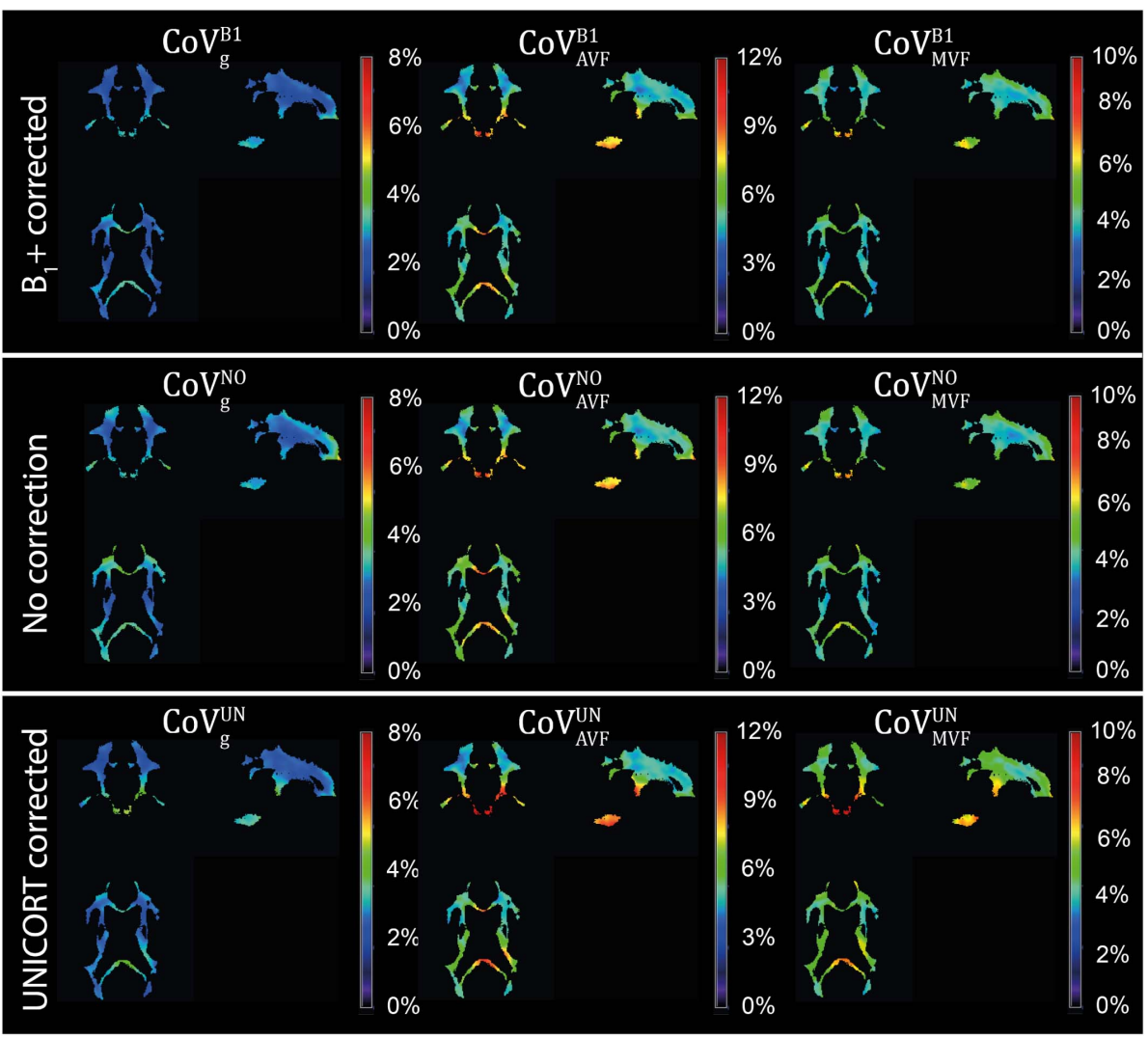

FIGURE 10 | Coefficient of variation (CoV) maps of $\mathrm{gMR}_{\mathrm{MR}}, \mathrm{AVF}_{\mathrm{MR}}$, and $\mathrm{MVF}_{\mathrm{MR}}$ with $\mathrm{B}_{1}+$ correction $\left(\mathrm{CoV}_{\mathrm{g}}^{\mathrm{B1}}, \mathrm{CoV}_{\mathrm{AVF}}^{\mathrm{B1}}\right.$, and $\left.\mathrm{CoV}_{\mathrm{MVF}}^{\mathrm{B1}}\right)$, no correction $\left(\mathrm{CoV} V_{\mathrm{g}}^{\mathrm{NO}}, \mathrm{CoV}_{\mathrm{AVF}}^{\mathrm{NO}}\right.$, and $\mathrm{CoV}_{\mathrm{MVF}}^{\mathrm{NO}}$ ), and UNICORT $\mathrm{B}_{1}+$ correction $\left(\mathrm{CoV}_{\mathrm{g}}^{\mathrm{UN}}\right.$, $\mathrm{CoV}_{\mathrm{AVF}}^{\mathrm{UN}}$, and $\left.\mathrm{CoV}_{\mathrm{MVF}}^{\mathrm{UN}}\right)$. CoV maps, expressed in percentage, were computed as the voxel-wise ratio between the group mean and group standard deviation maps of the normalized gMR, $A V F_{M R}$, or MVF $F_{M R}$. The voxel-wise computation of CoV is restricted to the group WM mask (cf. section "Definition of White Matter Masks"). Shown are a single coronal ( $y=91)$, sagittal $(x=100)$, and axial $(z=85)$ slice.

relevance for group studies because it can be regarded as an error that evolves when replacing the reference method with the alternative method. For comparison, age-related changes assessed by g-ratio weighted imaging (Cercignani et al., 2017; Berman et al., 2018) have been reported to vary between 30 and 100\% (in absolute values: $\mathrm{g}_{\mathrm{MR}} 0.02-0.04$ (Figure 5 in Cercignani et al., 2017). Consequently, the reported effect size of age-related changes would have become potentially undetectable if the $\mathrm{B}_{1}+$ field correction has been omitted in the study of Cercignani et al. (2017). The $B_{1}+$ effect is particularly relevant for the MR g-ratio method by Cercignani et al. (2017) that combined quantitative MT (Gloor et al., 2008) with NODDI, because the qMT method does not possess an intrinsic correction for $\mathrm{B}_{1}+$ field inhomogeneities as opposed to the $\mathrm{MT}_{\text {sat }}$ methods used here. Note that we reported, for better intuition, the bias and error relative to the dynamic range of the parameters across the investigated white matter (WM) ROIs (the dynamic range of $\mathrm{g}_{\mathrm{MR}}$ is $\triangle_{\mathrm{DR}}=0.046$; the absolute bias and error can be found in Table 4).

To reduce this source of bias and error, we propose a datadriven approach to correct for $\mathrm{B}_{1}+$ field inhomogeneities when no $B_{1}+$ field measurement is available. To this end, we used UNICORT to estimate the $B_{1}+$ field (Weiskopf et al., 2011). We found that using the UNICORT-estimated $\mathrm{B}_{1}+$ field to correct residual $\mathrm{B}_{1}+$ field inhomogeneities in $\mathrm{MT}_{\text {sat }}$ reduces at the group level the bias and error in the MR g-ratio and its constituents by roughly a factor of three. However, the UNICORT estimated $\mathrm{B}_{1}+$ inhomogeneity can be erroneous with the error varying across subjects. To assess this variability, we estimated coefficientof-variance $(\mathrm{CoV})$ maps of $\mathrm{g}_{\mathrm{MR}}, \mathrm{AVF}_{\mathrm{MR}}$, and $\mathrm{MVF}_{\mathrm{MR}}$ for all

TABLE 5 | Bias and error between methods, in the CoV of $\mathrm{g}_{\mathrm{MR}}, \mathrm{AVF}_{\mathrm{MR}}$, and MVFMR.

\begin{tabular}{|c|c|c|c|c|}
\hline MAP & $\bar{\delta}^{\mathrm{Cov}}$ & $\epsilon^{\mathrm{CoV}}$ & $\frac{\bar{\delta}^{\mathrm{CoV}}}{\mathrm{CoV}_{\mathrm{MR}}^{\mathrm{B1}}} \cdot 100$ & $\frac{\epsilon^{\mathrm{CoV}}}{\mathrm{CoV}_{\mathrm{MR}}^{\mathrm{B1}}} \cdot 100$ \\
\hline Cov g $g_{M R}^{B 1}$ vs. Cov g ${ }_{M R}^{N O}$ & -0.42 & 0.56 & -17.3 & 23.1 \\
\hline Cov g g1 vs. Cov g $g_{M R}^{\mathrm{BN}}$ & -0.12 & 0.62 & -4.9 & 25.5 \\
\hline CoV AVF ${ }_{M R}^{B 1}$ vs. CoV AVF ${ }_{M R}^{N O}$ & -0.40 & 0.78 & -7.3 & 14.3 \\
\hline CoV AVF ${ }_{M R}^{B 1}$ vs. CoV AVF $F_{M R}^{U N}$ & -0.21 & 1.02 & -3.8 & 18.7 \\
\hline CoV MVF ${ }_{M R}^{B 1}$ vs. CoV MVF ${ }_{M R}^{N O}$ & -0.05 & 0.41 & -1.1 & 9.2 \\
\hline CoV MVF ${ }_{M R}^{B 1}$ vs. CoV MVF $F_{M R}^{U N}$ & -0.52 & 1.20 & -11.9 & 27.0 \\
\hline
\end{tabular}

List of the bias ( $\bar{\delta}^{\mathrm{CoV}}$ ) and error $\left(\epsilon^{\mathrm{CoV}}\right)$ values as defined in Figure 11, along with their relative value with respect to the group-average CoV across the MR g-ratios using the reference $B_{1}+$ field correction method: $\frac{\bar{\delta}^{\operatorname{CoV}}}{\operatorname{CoV}_{M R}^{B 1}} \cdot 100 ; \frac{\epsilon^{\operatorname{CoV}}}{\operatorname{CoV}_{M R}^{B 1}} \cdot 100$. 

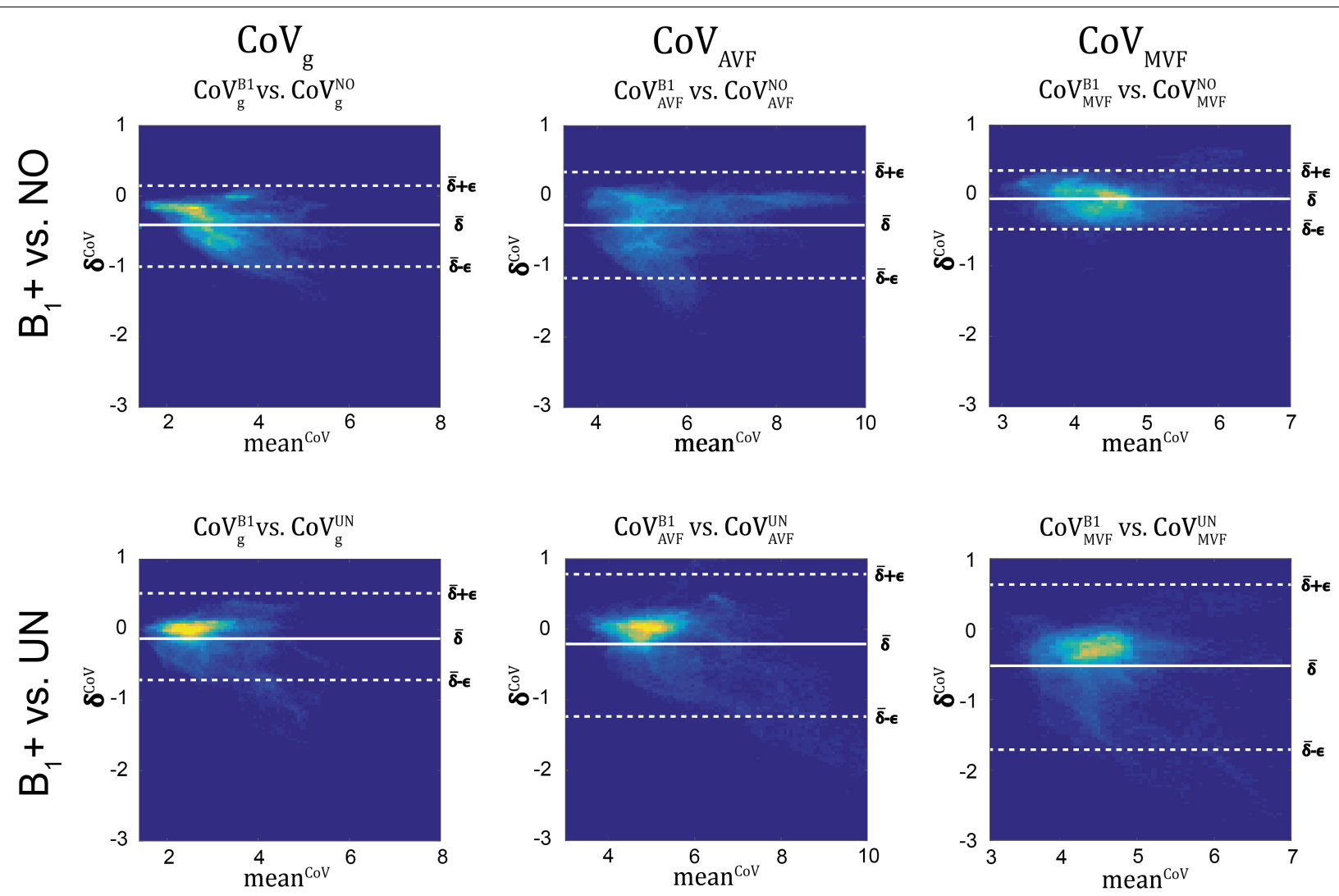

FIGURE 11 | Bland-Altman density plots of $\mathrm{CoV}_{\mathrm{g}}$, $\mathrm{CoV}_{\mathrm{AVF}}$, and $\mathrm{CoV}_{\mathrm{MVF}}$ for no correction (NO, top row) and UNICORT B + correction (UN, bottom row) against the reference method $\left(\mathrm{B}_{1}+\right.$ ) (yellow indicates high density and blue low). The Bland-Altman plot depicts the differences between the tested parameter maps and the reference method (e.g., $\left.\delta_{i}^{\mathrm{CoV}}=\left(\mathrm{CoV}_{\mathrm{g}}^{\mathrm{B} 1}\right)_{i}-\left(\mathrm{CoV}_{\mathrm{g}}^{\mathrm{k}}\right)_{i}\right)$ against their mean (e.g., mean $\left.\mathrm{CoV}_{i}=\frac{\left.\left(\mathrm{CoV}_{\mathrm{g}}^{\mathrm{B} 1}\right)_{i}+\mathrm{CoV}_{\mathrm{g}}^{\mathrm{k}}\right)_{i}}{2}\right)$ with $\mathrm{k}=\{\mathrm{UN}, \mathrm{NO}\}$ and $i$ being the index of the $i^{\text {th }}$ region. The bold white line represents the bias $\left(\bar{\delta}^{\mathrm{CoV}}=\sum_{i=1}^{N}{ }_{i}{ }^{\mathrm{CoV}} ; \mathrm{N}=\right.$ number of voxels) and the dashed lines represent $\bar{\delta}^{\mathrm{CoV}}$ the error $\left(\epsilon^{\mathrm{CoV}}=1.96 \cdot \mathrm{SD}\left(\delta_{i}^{\mathrm{CoV}}\right)\right]$. The error and bias values are summarized in Table 5 .

three methods. In general, an increased $\mathrm{CoV}$ can be found at tissue boundaries (e.g., cerebral spinal fluid to WM) due to slight misregistration between the maps of axonal and myelin markers and/or imperfect normalization (Figure 10). Additionally, we found a strong increase in the bias and error of the CoV of MVF maps (increase in bias: $11 \%$ and in error: $18 \%$ ) when UNICORT $\mathrm{B}_{1}+$ correction was used as compared to no correction. The $\mathrm{CoV}$ of $g_{M R}$ and $A_{V F} F_{M R}$ did not show a consistent trend: while the bias decreased, the error increased for both parameters. In other words, the UNICORT $\mathrm{B}_{1}+$ correction leads to higher accuracy in the g-ratio and its constituents but comes at the cost of a lower precision in MVF.

\section{G-ratio, Myelin, and Axonal Volume Fraction Across the White Matter}

Our $\mathrm{g}_{\mathrm{MR}}^{\mathrm{B} 1}$ and $\mathrm{AVF}_{\mathrm{MR}}^{\mathrm{B} 1}$ across the white matter were within the range of the reported values of previous studies ( $\mathrm{g}_{\mathrm{MR}}$ : 0.64-0.76; $\mathrm{AVF}_{\mathrm{MR}}$ : $0.26-0.43$ in (Cercignani et al., 2017; Berman etal., 2018). The range of $\mathrm{MVF}_{\mathrm{MR}}^{\mathrm{B1}}$ was in the upper half of previously reported values $(0.17-0.42$ in Cercignani et al., 2017). Our slightly higher $M_{\text {VF }}$ values might be due to differences in the calibration approach: while we calculated the reference $\mathrm{MVF}_{\mathrm{REF}}$ from previously published ex-vivo histology data (Graf von Keyserlingk and Schramm, 1984), Cercignani et al. (2017), used a reference from previously published ex-vivo histology g-ratio data in the corpus callosum and Berman et al. (2018), did not perform any calibration assuming that macromolecular tissue volume and $\mathrm{MVF}_{\mathrm{MR}}$ are equal. An error in the calibration constant can lead to a bias in the MVF estimates which in turn leads to an error and bias in the MR g-ratio (Campbell et al., 2018).

\section{Confounding Factors}

As this study calculates the in-vivo MR g-ratio, there is no histological data available from the participants of this study, which could be used for calibration or as a gold standard reference. For calibration of $\mathrm{MT}_{\text {sat }}$ to $\mathrm{MVF}_{\mathrm{MR}}$, we estimated the histological MVF ( $\left.\mathrm{MVF}_{\text {hist }}\right)$ from published ex-vivo data within the human medulla oblongata (Graf von Keyserlingk and Schramm, 1984). Since the reference $\mathrm{MVF}_{\text {hist }}$ and the calibrated $\mathrm{MT}_{\text {sat }}$ map were taken from different subjects, this might introduce a systematic bias in the MR g-ratio. However, since we found a relatively good agreement between our gMr, 


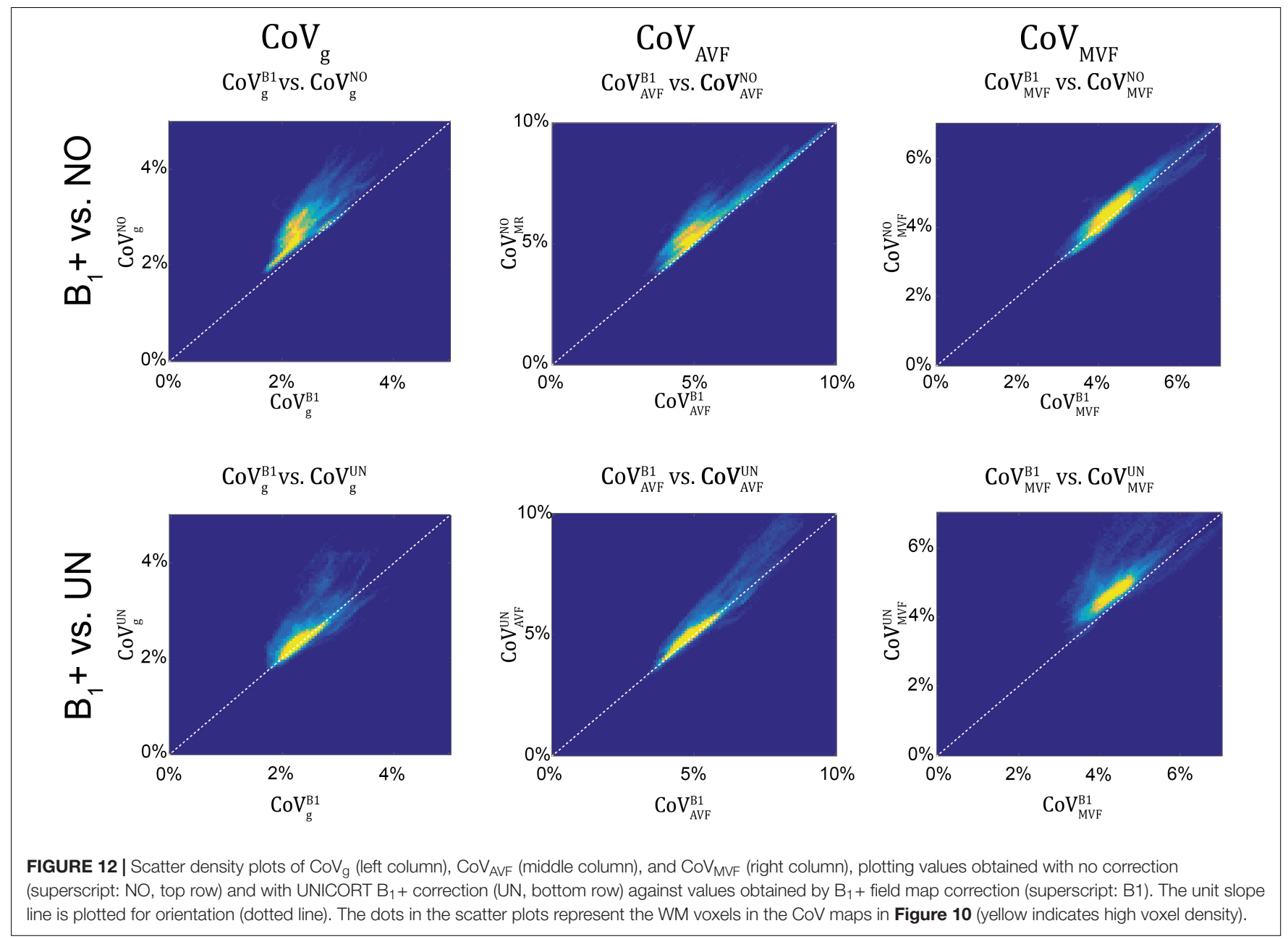

$\mathrm{AVF}_{\mathrm{MR}}$, and $\mathrm{MVF}_{\mathrm{MR}}$ values with previously reported values obtained by a different calibration approach (Cercignani et al., 2017; Berman et al., 2018), we expect that it had a small effect on the results. Moreover, we focused on the effect of omitting $\mathrm{B}_{1}+$ correction, which will lead to additional inaccuracies in g-ratio weighted imaging, independent of the quality of the calibration.

Although, not reported in previous NODDI-based g-ratio mapping studies (Stikov et al., 2015; Cercignani et al., 2017; Jung et al., 2017; Mancini et al., 2017; Ellerbrock and Mohammadi, 2018; Hori et al., 2018), we found that the intra-cellular volume fraction $\left(v_{\text {icvf }}\right)$ determined with NODDI tends to be biased at small signal-to-noise ratios $(\mathrm{SNR}<39)$, resulting in a ceiling effect, i.e., $\nu_{\text {icvf }} \approx 1$. To avoid a corresponding bias in $\mathrm{g}_{\mathrm{MR}}$ (and $\mathrm{AVF}_{\mathrm{MR}}$ ), we restricted the analysis to regions with sufficiently high SNR (Figure 3). To investigate whether our findings generalize to low-SNR regions as well, we performed an additional Bland-Altman analysis of $\mathrm{MVF}_{\mathrm{MR}}$ in whole-WM ROIs. To this end, a larger set of ROIs was used covering the entire white matter. Although the bias was smaller for the wholeWM as compared to the high-SNR ROI analysis, we found the same trend: the error and bias were reduced when using
UNICORT $\mathrm{B}_{1}+$ correction relative to no correction. Note that the smaller bias for the whole-WM analysis is most probably an artifact of the calibration procedure. Since the ROI used for calibration was not part of the high-SNR ROIs but was part of the whole-WM ROIs, we think it could have reduced the bias in the whole-WM ROI analysis as compared to the highSNR analysis.

We note that the presented results were based on a customized $\mathrm{B}_{1}+$ mapping method (Lutti et al., 2010). Using vendor specific protocols for $\mathrm{B}_{1}+$ and $\mathrm{MT}_{\text {sat }}$ mapping may influence the results (Leutritz et al., 2020). Moreover, the calibration factor in Equation (1) may have to be recalibrated for different MT-pulses.

Future studies should investigate the effect of $\mathrm{B}_{1}+$ correction on MR g-ratio mapping when using alternative biomarkers to estimate $\mathrm{AVF}_{\mathrm{MR}}$ and $\mathrm{MVF}_{\mathrm{MR}}$ (e.g., Ellerbrock and Mohammadi, 2018). Moreover, there are alternative $B_{1}+$ mapping approaches available which might vary in precision (Lutti et al., 2010) and therefore can affect the MR g-ratio values. However, the differences in the precision of these methods are in the order of few percentage and thus much smaller than the effect of omitting the $\mathrm{B}_{1}+$ field or using the data-driven UNICORT $\mathrm{B}_{1}+$ estimate (Weiskopf et al., 2011). 


\section{CONCLUSION}

In this study, we assessed the effect of $\mathrm{B}_{1}+$ correction on the accuracy of MR g-ratio as well as axonal and myelin volume fraction based on $\mathrm{MT}_{\text {sat }}$ and NODDI. Our results demonstrate that $\mathrm{B}_{1}+$ correction via a measured $\mathrm{B}_{1}+$ field map is the method of choice. If the $\mathrm{B}_{1}+$ field map cannot be acquired, we propose the retrospective, data-driven UNICORT $\mathrm{B}_{1}+$ correction to estimate and correct for $\mathrm{B}_{1}+$ field inhomogeneities, which reduces the error and bias by a factor of three. UNICORT is implemented in the free and open-source hMRI toolbox (see text footnote 1).

\section{DATA AVAILABILITY STATEMENT}

The datasets presented in this article are not readily available because the data that support the findings of this study are available on request from the corresponding author. The data have not been made freely available on the internet due to privacy or ethical restrictions. Requests to access the datasets should be directed to corresponding author.

\section{ETHICS STATEMENT}

The studies involving human participants were reviewed and approved by the Ärztekammer Hamburg. The patients/participants provided their written informed consent to participate in this study.

\section{AUTHOR CONTRIBUTIONS}

SM and TE contributed to the conception and design of the study, performed statistical analysis and MRI processing, and wrote the

\section{REFERENCES}

André, E. D., Grinberg, F., Farrher, E. I, Maximov, I., Shah, N. J., Meyer, C., et al. (2014). Influence of noise correction on intra- and inter-subject variability of quantitative metrics in diffusion kurtosis imaging. PLoS One 9:e94531. doi: 10.1371/journal.pone.0094531

Ashburner, J. (2007). A fast diffeomorphic image registration algorithm. Neuroimage 38, 95-113. doi: 10.1016/j.neuroimage.2007.07.007

Berman, S., West, K. L., Does, M. D., Yeatman, J. D., and Mezer, A. A. (2018). Evaluating g-ratio weighted changes in the corpus callosum as a function of age and sex. Neuroimage 182, 304-313. doi: 10.1016/j.neuroimage.2017.06.076

Bland, J. M., and Altman, D. G. (1986). Statistical methods for assessing agreement between two methods of clinical measurement. Lancet 1, 307-310. doi: 10.1128/ AAC.00483-18

Callaghan, M. F., Helms, G., Lutti, A., Mohammadi, S., and Weiskopf, N. (2015a). A general linear relaxometry model of R1 using imaging data. Magn. Reson. Med. 73, 1309-1314. doi: 10.1002/mrm.25210

Callaghan, M. F., Josephs, O., Herbst, M., Zaitsev, M., Todd, N., and Weiskopf, N. (2015b). An evaluation of prospective motion correction (PMC) for high resolution quantitative MRI. Front. Neurosci. 9:97. doi: 10.3389/fnins.2015. 00097

Callaghan, M. F., Lutti, A., Ashburner, J., Balteau, E., Corbin, N., Draganski, B., et al. (2019). Example dataset for the hMRI toolbox. Data Brief 25:104132. doi: 10.1016/j.dib.2019.104132 first draft of the manuscript. All authors contributed substantially to revising the manuscript critically for intellectual content and have approved the submitted version.

\section{FUNDING}

This project was by the ERA-NET NEURON (hMRIofSCI), the Federal Ministry of Education and Research (BMBF; 01EW1711A and B), and the German Research Foundation (DFG Priority Program 2041 "Computational Connectomics," (AL 1156/21;GE 2967/1-1; MO 2397/5-1; MO 2249/3-1), DFG Emmy Noether Stipend: MO 2397/4-1), and the Forschungszentrums Medizintechnik Hamburg (fmthh; grant 01fmthh2017). GH was supported by the Swedish Research Council (NT 20146193). PF was funded by a SNF Eccellenza Professorial Fellowship grant (PCEFP3_181362/1), and the European Union's Horizon 2020 research, innovation programme (grant agreement no 634541). EB received funding from the European Structural and Investment Fund/European Regional Development Fund and the Belgian Walloon Government, project BIOMED-HUB (programme 20142020). Our gratitude extends also to Jürgen Finsterbusch for support on the MR side, to the University of Minnesota Centre for Magnetic Resonance Research for providing the image reconstruction algorithm for the simultaneous multislice acquisitions.

\section{SUPPLEMENTARY MATERIAL}

The Supplementary Material for this article can be found online at: https://www.frontiersin.org/articles/10.3389/fnins. 2021.674719/full\#supplementary-material

Campbell, J. S. W., Leppert, I. R., Boudreau, M., Narayanan, S., Duval, T., CohenAdad, J., et al. (2018). Promise and pitfalls of g-ratio estimation with MRI. Neuroimage 182, 80-96. doi: 10.1016/j.neuroimage.2017.08.038

Cercignani, M., Giulietti, G., Dowell, N. G., Gabel, M., Broad, R., Leigh, P. N., et al. (2017). Characterizing axonal myelination within the healthy population: a tract-by-tract mapping of effects of age and gender on the fiber g-ratio. Neurobiol. Aging 49, 109-118. doi: 10.1016/j.neurobiolaging.2016.09.016

Ellerbrock, I., and Mohammadi, S. (2018). Four in vivo g -ratio-weighted imaging methods: comparability and repeatability at the group level. Hum. Brain Mapp. 39, 24-41. doi: 10.1002/hbm.23858

Feinberg, D. A., Moeller, S., Smith, S. M., Auerbach, E., Ramanna, S., Glasser, M. F., et al. (2010). Multiplexed echo planar imaging for sub-second whole brain FMRI and Fast Diffusion Imaging. Edited by Pedro Antonio Valdes-Sosa. PLoS One 5:e15710. doi: 10.1371/journal.pone.0015710

Gloor, M., Scheffler, K., and Bieri, O. (2008). Quantitative magnetization transfer imaging using balanced SSFP. Magn. Reson. Med. 60, 691-700. doi: 10.1002/ mrm. 21705

Graf von Keyserlingk, D., and Schramm, U. (1984). Diameter of axons and thickness of myelin sheaths of the pyramidal tract fibres in the adult human medullary pyramid. Anat. Anz. 157, 97-111. doi: 10.1097/PSY. 0000000000000183

Helms, G. (2015). "Correction for residual effects of B1+ inhomogeniety on MT saturation in FLASH-based multi-parameter mapping of the brain," in Proceedings of the International Society for Magnetic Resonance in Medicine 
Annual Meeting 2015, (Toronto: International Society for Magnetic Resonance in Medicine), 3360.

Helms, G., Dathe, H., Kallenberg, K., and Dechent, P. (2008). High-resolution maps of magnetization transfer with inherent correction for RF inhomogeneity and T 1 relaxation obtained from 3D FLASH MRI. Magn. Reson. Med. 60, 1396-1407. doi: 10.1002/mrm.21732

Helms, G., Weiskopf, N., and Lutti, A. (2021). Correction of FLASH-based MT saturation in human brain for residual bias of B1-inhomogeneity at 3T. arXiv [Preprint]. arXiv:210414878 [physics].

Hildebrand, C., and Hahn, R. (1978). Relation between myelin sheath thickness and axon size in spinal cord white matter of some vertebrate species. J. Neurol. Sci. 38, 421-434. doi: 10.1016/0022-510X(78)90147-8

Hori, M., Hagiwara, A., Fukunaga, I., Ueda, R., Kamiya, K., Suzuki, Y., et al. (2018). Application of quantitative microstructural MR imaging with atlasbased analysis for the spinal cord in cervical spondylotic myelopathy. Sci. Rep. 8, 1-8. doi: 10.1038/s41598-018-23527-8

Hua, K., Zhang, J., Wakana, S., Jiang, H., Li, X., Reich, D. S., et al. (2008). Tract probability maps in stereotaxic spaces: analyses of white matter anatomy and tract-specific quantification. Neuroimage 39, 336-347. doi: 10.1016/j. neuroimage.2007.07.053

Jung, W., Lee, J., Shin, H. G., Nam, Y., Zhang, H., Oh, S. H., et al. (2017). Whole brain g-ratio mapping using myelin water imaging (MWI) and neurite orientation dispersion and density imaging (NODDI). Neuroimage 19, 109-118. doi: 10.1016/j.neuroimage.2017.09.053

Kamagata, K., Zalesky, A., Yokoyama, K., Andica, C., Hagiwara, A., Shimoji, K., et al. (2019). MR g-ratio-weighted connectome analysis in patients with multiple sclerosis. Sci. Rep. 9:13522. doi: 10.1038/s41598-019-50025-2

LaMantia, A.-S., and Rakic, P. (1990). Cytological and quantitative characteristics of four cerebral commissures in the rhesus monkey. J. Comp. Neurol. 291, 520-537. doi: 10.1002/cne.902910404

Lehmenkühler, A., Syková, E., Svoboda, J., Zilles, K., and Nicholson, C. (1993). Extracellular space parameters in the rat neocortex and subcortical white matter during postnatal development determined by diffusion analysis. Neuroscience 55, 339-351. doi: 10.1016/0306-4522(93)90503-8

Leutritz, T., Seif, M., Helms, G., Samson, R. S., Curt, A., Freund, P., et al. (2020). Multiparameter mapping of relaxation (R1, R2*), proton density and magnetization transfer saturation at $3 \mathrm{~T}$ : a multicenter dual-vendor reproducibility and repeatability study. Hum. Brain Mapp. 41, 4232-4247. doi: 10.1002/hbm. 25122

Liewald, D., Miller, R., Logothetis, N., Wagner, H.-J., and Schüz, A. (2014). Distribution of axon diameters in cortical white matter: an electronmicroscopic study on three human brains and a macaque. Biol. Cybern. 108, 541-557. doi: 10.1007/s00422-014-0626-2

Lutti, A., Hutton, C., Finsterbusch, J., Helms, G., and Weiskopf, N. (2010). Optimization and validation of methods for mapping of the radiofrequency transmit field at 3T. Magn. Reson. Med. 238, 229-238. doi: 10.1002/mrm.22421

Macdonald, J., and Ruthotto, L. (2018). Improved susceptibility artifact correction of echo-planar MRI using the alternating direction method of multipliers. J. Math. Imaging Vis. 60, 268-282. doi: 10.1007/s10851-017-0757-X

Mancini, M., Giulietti, G., Dowell, N., Spanò, B., Harrison, N., Bozzali, M., et al. (2017). Introducing axonal myelination in connectomics: a preliminary analysis of g-ratio distribution in healthy subjects. Neuroimage 182, 351-359. doi: 10 . 1016/j.neuroimage.2017.09.018

Moeller, S., Yacoub, E., Olman, C. A., Auerbach, E., Strupp, J., Harel, N., et al. (2010). Multiband multislice GE-EPI at 7 tesla, with 16-fold acceleration using partial parallel imaging with application to high spatial and temporal whole-brain FMRI. Magn. Reson. Med. 63, 1144-1153. 10.1002/mrm. 22361

Mohammadi, S., and Callaghan, M. F. (2020). Towards in vivo g-ratio mapping using MRI: unifying myelin and diffusion imaging. J. Neurosci. Methods 348:108990. doi: 10.1016/j.jneumeth.2020.108990

Mohammadi, S., Carey, D., Dick, F., Diedrichsen, J., Sereno, M. I., Reisert, M., et al. (2015). Whole-brain in-vivo measurements of the axonal g-ratio in a group of 37 healthy volunteers. Front. Neurosci. 9:441. doi: 10.3389/fnins.2015.00441

Mohammadi, S., Möller, H. E., Kugel, H., Müller, D. K., and Deppe, M. (2010). Correcting eddy current and motion effects by affine whole-brain registrations: evaluation of three-dimensional distortions and comparison with slicewise correction. Magn. Reson. Med. 64, 1047-1056. doi: 10.1002/mrm. 22501

Mohammadi, S., Tabelow, K., Ruthotto, L., Feiweier, T., Polzehl, J., and Weiskopf, N. (2014). High-resolution diffusion kurtosis imaging at 3t enabled by advanced post-processing. Front. Neurosci. 8:427. doi: 10.3389/fnins.2014.00427

Nicholson, C., and Hrabitová, S. (2017). Brain extracellular space: the final frontier of neuroscience. Biophys. J. 113, 2133-2142. doi: 10.1016/j.bpj.2017.06.052

Olivares, R., Montiel, J., and Aboitiz, F. (2001). Species differences and similarities in the fine structure of the mammalian corpus callosum. Brain Behav. Evol. 57, 98-105. doi: 10.1159/000047229

Reese, T. G., Heid, O., Weisskoff, R. M., and Wedeen, V. J. (2003). Reduction of eddy-current-induced distortion in diffusion MRI using a twice-refocused spin echo. Magn. Reson. Med. 49, 177-182. doi: 10.1002/mrm.10308

Ruthotto, L., Kugel, H., Olesch, J., Fischer, B., Modersitzki, J., Burger, M., et al. (2012). Diffeomorphic susceptibility artifact correction of diffusion-weighted magnetic resonance images. Phys. Med. Biol. 57, 5715-5731. doi: 10.1088/0031$9155 / 57 / 18 / 5715$

Ruthotto, L., Mohammadi, S., Heck, C., Modersitzki, J., and Weiskopf, N. (2013). "Hyperelastic susceptibility artifact correction of DTI in SPM," in Bildverarbeitung Für Die Medizin 2013, eds H. P. Meinzer, T. Deserno, H. Handels, and T. Tolxdorff (Berlin: Springer), 344-349. doi: 10.1007/978-3-64236480-8_60

Sereno, M. I., Lutti, A., Weiskopf, N., and Dick, F. (2013). Mapping the human cortical surface by combining quantitative T1 with retinotopy. Cereb. Cortex 23, 2261-2268. doi: 10.1093/cercor/bhs213

Setsompop, K., Gagoski, B. A., Polimeni, J. R., Witzel, T., Wedeen, V. J., and Wald, L. L. (2012). Blipped-controlled aliasing in parallel imaging for simultaneous multislice echo planar imaging with reduced g-factor penalty. Magn. Reson. Med. 67, 1210-1224. doi: 10.1002/mrm.23097

Stikov, N., Campbell, J. S., Stroh, T., Lavelée, M., Frey, S., Novek, J., et al. (2015). In vivo histology of the myelin g-ratio with magnetic resonance imaging. Neuroimage 118, 397-405. doi: 10.1016/j.neuroimage.2015.05.023

Stikov, N., Perry, L. M., Mezer, A., Rykhlevskaia, E., Wandell, B. A., Pauly, J. M., et al. (2011). Bound pool fractions complement diffusion measures to describe white matter micro and macrostructure. Neuroimage 54, 1112-1121. doi: 10. 1016/j.neuroimage.2010.08.068

Swadlow, H. A., Waxman, S. G., and Geschwind, N. (1980). Small-diameter nonmyelinated axons in the primate corpus callosum. Arch. Neurol. 37, 114115. doi: 10.1001/archneur.1980.00500510072016

Tabelow, K., Balteau, E., Ashburner, J., Callaghan, M. F., Draganski, B., Helms, G., et al. (2019). hMRI - A toolbox for quantitative MRI in neuroscience and clinical research. Neuroimage 194, 191-210.

Tønnesen, J., Inavalli, V. V. G. K., and Nägerl, U. V. (2018). Super-resolution imaging of the extracellular space in living brain tissue. Cell 172, 1108-1121. doi: 10.1016/j.cell.2018.02.007

Wang, S. S., Shultz, J. R., Burish, M. J., Harrison, K. H., Hof, P. R., Towns, L. C., et al. (2008). Functional trade-offs in white matter axonal scaling. J. Neurosci. 28, 4047-4056. doi: 10.1523/JNEUROSCI.5559-05.2008

Weiskopf, N., Lutti, A., Helms, G., Novak, M., Ashburner, J., and Hutton, C. (2011). Unified segmentation based correction of R1 brain maps for RF transmit field inhomogeneities (UNICORT). Neuroimage 54, 2116-2124. doi: 10.1016/j. neuroimage.2010.10.023

Weiskopf, N., Suckling, J., Williams, G., Correia, M. M., Inkster, B., Tait, R., et al. (2013). Quantitative multi-parameter mapping of R1, PD(*), MT, and R2(*) at 3T: a multi-center validation. Front. Neurosci. 7:95. doi: 10.3389/fnins.2013. 00095

West, K. L., Kelm, N. D., Carson, R. P., and Does, M. D. (2016). A revised model for estimating g-ratio from MRI. Neuroimage 125, 1155-1158. doi: 10.1016/j. neuroimage.2015.08.017

Xu, J., Moeller, S., Auerbach, E. J., Strupp, J., Smith, S. M., Feinberg, D. A., et al. (2013). Evaluation of slice accelerations using multiband echo planar imaging at 3T. Neuroimage 83, 991-1001. doi: 10.1016/j.neuroimage.2013.07. 055

Yu, F., Fan, Q., Tian, Q., Ngamsombat, C., Machado, N., Bireley, J. D., et al. (2019). Imaging g-ratio in multiple sclerosis using high-gradient diffusion mri and macromolecular tissue volume. AJNR Am. J. Neuroradiol. 40, 1871-1877. doi: 10.3174/ajnr.A6283 
Zhang, H., Schneider, T., Wheeler-Kingshott, C. A., and Alexander, D. C. (2012). NODDI: practical in vivo neurite orientation dispersion and density imaging of the human brain. Neuroimage 61, 1000-1016. doi: 10.1016/j.neuroimage.2012. 03.072

Conflict of Interest: The authors declare that the research was conducted in the absence of any commercial or financial relationships that could be construed as a potential conflict of interest.
Copyright (c) 2021 Emmenegger, David, Ashtarayeh, Fritz, Ellerbrock, Helms, Balteau, Freund and Mohammadi. This is an open-access article distributed under the terms of the Creative Commons Attribution License (CC BY). The use, distribution or reproduction in other forums is permitted, provided the original author(s) and the copyright owner(s) are credited and that the original publication in this journal is cited, in accordance with accepted academic practice. No use, distribution or reproduction is permitted which does not comply with these terms. 